\section{International Scientific Journal Theoretical \& Applied Science}

p-ISSN: 2308-4944 (print) e-ISSN: 2409-0085 (online)

Year: 2017 Issue: 08 Volume: 52

Published: $14.08 .2017 \quad$ http://T-Science.org

\section{S.U. Zhanatauov}

candidate of physics and mathematical sciences, Department «Automation and information technologies»), Associate professor, Noncommercial joint-stock company "Kazakh national agrarian university" Kazakhstan sapagtu@mail.ru

SECTION 31. Economic research, finance, innovation, risk management.

\title{
A MODEL OF CALCULATION RISK CHANGING OF THE INTEREST RATE "YIELD TO MATURITY DATE" FOR FOREIGN CURRENCY BONDS OF THE REPUBLIC OF KAZAKHSTAN
}

Abstract: The work is devoted to analysis of real data on yields of foreign currency government securities $(G S)$ of the Republic of Kazakhstan: a fixed interest rate type - "yield to maturity date", developed a model using gauges degrees of volatility and risk changes yield GS are calculated (in\%) interest rate risk values of the form "risk of changes in the yield to maturity date" for high-risk (for Criteria 1 and 2) financial instruments (FI). Table "object property" $X_{131,6}^{\circ}$, yield values of the nodes 6 intervals yield curve temporal structure is interpreted as a multidimensional sample of the general population with an unknown probability distribution. Using principal component analysis in process performance for multivariate data solved the problem of allocation of 6 independent combinations of new financial instruments (NFI). Resolves an almost essential for banks to task allocation (by the Diekmann-Kaiser criterion) 3 local high-risk portfolios: NFI №1, NFI №2, NFI №3, each marked by the NFI Criteria 1 and 2, and calculated almost "visible" changes the values of yield risk to the date of maturity" for highrisk $F I$.

Key words: yield curve, yield to maturity date, time structure of interest rates, principal component analysis.

Language: Russian

Citation: Zhanatauov SU (2017) A MODEL OF CALCULATION RISK CHANGING OF THE INTEREST RATE "YIELD TO MATURITY DATE" FOR FOREIGN CURRENCY BONDS OF THE REPUBLIC OF KAZAKHSTAN. ISJ Theoretical \& Applied Science, 08 (52): 19-36.

Soi: http://s-o-i.org/1.1/TAS-08-52-4 Doi: crossef https://dx.doi.org/10.15863/TAS.2017.08.52.4

\section{МОДЕЛЬ РАСЧЕТА РИСКА ИЗМЕНЕНИЯ ПРОЦЕНТНОЙ СТАВКИ "ДОХОДНОСТЬ К ДАТЕ ПОГАШЕНИЯ" ДЛЯ ВАЛЮТНЫХ ОБЛИГАЦИЙ РЕСПУБЛИКИ КАЗАХСТАН}

\begin{abstract}
Аннотация: Работа посвящена анализу реальных данных по доходностям валютных ГЦБ Республики Казахстан: при фиксированном типе процентной ставки - «доходность к дате погашения», разработана модель, с использованием измерителей степеней волатильности и рисковости изменения доходностей ГЦБ вычислены (в \%-ах) значения прочентного риска вида "риск изменения доходности к дате погашения» для высокорисковых (по Критериям 1 и 2) финансовых инструментов (ФИ). Таблица "объект-свойство" $X_{131,6,}^{\circ}$ из значений доходностей в узлах 6 интервалов временной структуры кривой доходности интерпретируется как многомерная выборка из генеральной совокупности с неизвестным законом распределения вероятностей. С применением модели анализа главных компонент решена задача выделения 6 комбинаций независимых новых финансовых инструментов (НФИ). Решена практически важная для банков задача выделения (по критерию Дикмана-Кайзера) 3 высокорисковых локальных портфелей: НФИ №1, НФИ №2, НФИ №3, для каждого НФИ выделены по Критериям 1 и 2 и вычислены практически «заметные» значения рисков изменения доходности к дате погашения» для высокорисковых ФИ.

Ключевые слова: кривая доходности, доходность к дате погашения, временная структура процентных ставок, анализ главных компонент.
\end{abstract}

Введение.

В связи с стремлением Казахстана выпускать государственные ценные бумаги (ГЦБ) в иностранной валюте актуальны вопросы формирования цифровых данных по процентным ставкам типа «доходность к дате погашения» и

ISPC Technology and Innovation, 
извлечение из этих реальных данных конкретных «цифровых» знаний, практически полезных владельцу валютного портфеля ГЦБ. В ситуациях быстро изменяяющихся курсов валют, цен на акции и цен за 1 баррель нефти актуальны «узкие» интервалы изменения указанных ниже «числа дней до даты погашения». Хотя на бирже регистрируются доходности ГЦБ по международным стандартам, т.е. по частым и коротким интервалам времени, коммерческие банки вынуждены агрегировать (объединять) соседние временные интервалы, чтобы как-то сопоставлять их со сроками возврата краткосрочных кредитов. Одним из подходящих иллюстраций ответов на наш вопрос являются результаты анализа данных развивающегося фондового рынка РК за 1999 год на примере долларовых ГЦБ. В 1999 году положение долларовых ГЦБ в экономике РК аналогично статусу ГЦБ в валюте азиатской страны, которую (ГЦБ) Казахстан стремится выпускать в настоящем и в будущем. Ситуации на фондовом рынке РК в 90-ые годы и в настоящее время в рассматриваемом нами аспекте схожи.

Применяемая нами прямая модель главных компонент (ПМ ГК [1]), в основе которой лежит прямая задача анализа главных компонент (ПЗ АГК [2-4]) разработана Г.Хотеллингом [1]. ПМ ГК применялась успешно для выявления особенностей рынков ЦБ разных стран: Норвегия и ЕС [5], США, Германия, Япония, [6], Нидерланды $[7,8,9]$. В инвестиционной политике европейских банков [8] возросли их интересы к валютным (в \$) ГЦБ: число участников на их торгах возросли в разы. В активной политике банков их менеджеры применяют методы, основанные на манипулировании кривой доходности и на операции «swap» с ценными бумагами [10]. Банки особенно агрессивно настроены, когда возвраты по кредитам малы, но продажи ценных бумаг, рыночные цены которых возросли, увеличивают чистый доход и прибыль акционеров. Издержки по работе с ГЦБ снижают [11] чистый доход банка, хотя доходы от ГЦБ, купленных на торговой площадке Казахстанской фондовой биржы методом открытых торгов не облагаются налогом. Поэтому менеджеры не пойдут на подобные потери, пока не докажут Совету директоров, что издержки будут компенсированы более высоким ожидаемым доходом по каким-либо новым активам, приобретенным за счет поступлений от продаж валютных ГЦБ - весьма ценных финансовых инструментов (ФИ). Она - дисконтная облигация (ее цена меньше номинала) и на бирже эмитентом к аукциону вычисляются spot-ставки типа «доходность к дате погашения», а продажные цены повышаются с уменьшением spot-ставок [11-13].

\section{Кривая доходности.}

Функция зависимости вычисленных значений $\mathrm{x}_{\mathrm{j}}$ доходности (типа «доходность к дате погашения») от номера ј временного интервала изображается в виде графика и ее называют «кривая доходности». Номера интервалов упорядочены в порядке возрастания «сроков жизни ГЦБ». Упорядоченная цепочка интервалов «времен жизни до даты погашения ГЦБ» образует в нашей статье конкретную совокупность из 6 временных интервалов и называется «временной структурой процентных ставок» («доходности к дате погашения») в день инвестирования (в момент времени t). В один интервал «времени жизни» отбирают ГЦБ со схожими и практически идентичными характеристиками $[14,15]$.

Тип процентных ставок определяяет тип временной структуры, и в зависимости от типа ставок могут быть построены различные типы кривой доходности: кривая доходности к дате погашения, кривая бескупонной доходности, кривая форвардной ставки и мгновенной форвардной ставки. Преимущество выбранных нами типов ставки и риска состоит в их возможности сопоставлять (сравнивать) по степени рисковости и доходности ГЦБ, имеющих разные сроки до даты погашения. Это очень важное преимущество. Кроме того, используемые нами интервалы временной структуры применимы для вычисления изменения цены ГЦБ, выраженной в процентах, приблизительно равной скорректированной длительности (дюрации) ГЦБ, умноженной на изменение ее доходности к сроку погашения, зависящей от номера интервала. Мы ниже анализируем изменения доходностей, а не изменения цен ГЦБ $[12,13]$.

Анализ кривой доходности [9] дает возможность составить прогноз будущих изменений процентных ставок, а форма кривой доходности в «краткосрочных» интервалах их изменения дает банку ключ для выявления заниженной или завышенной оценки цены ГЦБ [12-14]. Если на графике кривой доходности поставить точку, характеризующую доходность новой ГЦБ, выше кривой, то эта ГЦБ представляет собой «привлекательный объект покупки», ибо значение ее доходности к дате погашения выше, а если эта точка лежит ниже кривой, то новая ГЦБ представляет собой возможный объект продажи или отказа от покупки. Мы не анализируем цены покупок и продаж ГЦБ, зависящих от кривой доходности. Но кривые доходности активно применяются при ценообразовании в методе процентных ожиданий: в операции «swap» - в обмене одних облигаций на другие при ожидании изменения 
процентных ставок или просто для обеспечения сиюминутного более высокого дохода. При этом учитывается как выигрыш от процентных платежей, так и разница курсов продавцов и покупателей. Еще одна разновидность «swap» обмен облигаций с неодинаковым риском изменения процентных ставок. Для проведения таких операций требуется, чтобы на рынке преобладали ненормально низкие или ненормально высокие ставки. Такая ситуация наблюдается, например, на вторичном рынке продаж негосударственных облигаций, когда покупатель использует еe, например, для включения в уставной фонд.

Используя кривую доходности нашего типа и вычисленные ниже риски ГЦБ, банк будет продавать (покупать) ценные бумаги, которые переоценены из-за низких процентных ставок, и ценные бумаги, которые недооценены из-за высоких ставок, а затем будет покупать (продавать) на вторичном рынке ценные бумаги, правильно оцененные рынком. Когда же на рынке наступит равновесие, банк осуществит обратные покупки (продажи) и получит прибыль.

Кривая доходности позволяет по некоторым признакам оценить величины будущих процентных ставок, показать изменения или тренды (вверх или вниз) доходностей ГЦБ в зависимости от сроков погашения. Например, рассмотрим ГЦБ, которая на предыдущем аукционе (срок к дате погашения равен 1 году, временной интервал №4) продавалась по цене, соответствующей «доходности к дате погашения», равной $6 \%$, а другая ГЦБ (со сроком погашения 2 года, временной интервал №5) продавалась по цене, соответствующей «доходности к дате погашения», равной $8 \%$. Такой разрыв по доходности может помочь инвестору узнать увеличение новой (будущей) величины «доходности к сроку погашения» на следующем, 2-ом году жизни ГЦБ. Такой разрыв по доходности может быть представлен кривой, имеющей положительный угол наклона. Такая уверенность инвестора отно сительно угла наклона кривой доходности приведет его к покупке «2-годичной» ГЦБ. При этом \$100 начальных инвестиций возрастут за 2 года до $\$ 116,64: \quad \$ 100(1+0.08)(1+0.008)=\$ 116,64$. Эта прибыль в \$16.64 будет достигнута через 2 года, если 1-годичная ГЦБ будет продана после накопленной цены: $\$ 100(1+0.06)=\$ 106$. Во 2-ом году при процентной ставке, равной $10.4 \%$. Доход этой ГЦБ (\$106), который будучи реинвестирован (через продажу ГЦБ) с доходностью в $10.4 \%$, даст дополнительный доход, что в итоге составит $\$ 116,64: \$ 100(1+0.06)(1+0.104)=$

$\$ 106(1+0.104)=\$ 116.64$. Это значение $10.4 \%$ представляет собой форвардную ставку (forward rate), которую увидел инвестор, используя спотставки (spot rate) 6\%, 8\%, и кривую доходности. Данный пример иллюстрирует «метод процентных ожиданий» в активной политике банка. Другие применения кривой доходности имеют ся в [5-13].

\section{Интервалы временной структуры кривой доходности.}

В статье рассмотрен один тип процентных ставок - «доходность к дате погаше ния» и рассчитаны значения процентных рисков изменения «доходности к дате погашения». Зависящих от сроков до даты погашения. Временных интервалов 6:

1) «количество дней до даты погашения меняется в интервале [8 дней - 30 дней];

2) «количество дней до даты погашения меняется в интервале [31 дней - 90 дней];

3) «количество дней до даты погашения меняется в интервале [91 дня - 180 дней];

4) «количество дней до даты погашения меняется в интервале [181 дней - 360 дней];

5) «количество дней до даты погашения меняется в интервале [361 день - 1080 дней];

6) «количество дней до даты погашения меняется в интервале [1081 и более дней].

Приведенная классификация ГЦБ (активов банка) по «времени жизни» ГЦБ соответствует банкам стран с развивающейся экономикой.

В мировой практике принята следующая класси фикация по степени срочности актива: «короткие» (до 30 дней), краткосрочные (от 1 месяца до 1 года); среднесрочные (от 1 года до 3 лет); долгосрочные (от 3-х лет и выше). В соответствии с этой классификацией приводимые в нашей статье интервалы временной структуры доходности типа «доходность к дате погашения» не соответствуют классификации активов по срочности. Обоснованным отличием является разбиение на 3 части краткосрочных активов: (от 1 месяца до 3 месяцев $)+$ (от 3 месяцев до полугода) + (от полугода до 1 года). Краткосрочные активы весьма привлекательны по доходности для банка и такое разбиение позволяет банку Республики Казахстан иметь очевидную выгоду: 3-кратная капитализация дохода выше 1-кратной капитализации. Например, получение дохода в $20 \%$ лучше проводить в 3 этапа по времени: если доходность в 20\% распределена на 3 даты их получения: $9+5+6=20$, то $\$ 100$ к 1 -ой дате даст доход в $\$ 109$, ко 2 -ой - $\$ 109 *(0.5)=\$ 114,45$, к 3-ей - $\$ 121,317=\$ 114,45+\$ 6,867$. Доход в $\$ 121,317$ превышает доход в $\$ 120$ при одноразовой капитализации.

\section{Описание исходных данных.}


Были предоставлены данные в виде таблицы (ЭТ EXCEL) из 14 столбцов, 5000 строк. Эта база данных формировалась из таблиц, отображающих результаты дневных торгов ГЦБ на фондовой бирже Республики Казахстан (KASE) за дни проведения аукционов. Число аукционов в неделю зависит от числа рабочих дней в неделю. Из этой таблицы годовых процентных ставок типа «доходность к дате погашения» валютных ГЦБ РК на фондовой бирже, классифицированных по ее столбцам по количеству дней до даты погашения, была сформирована полная таблица «объект-свойство» $\mathrm{X}^{0}{ }_{131,6}$ размерности 131 на 6 . Для і-го объекта свойством №j является значение $\mathrm{x}_{\mathrm{i}, \mathrm{j}}^{0}$ процентной ставки типа «доходность к дате погашения» у ГЦБ, срок жизни которой принадлежит ј-ому интервалу временной структуры. Если ј-ому интервалу в і-ый день аукциона соответствуют несколько значений $\mathrm{x}_{\mathrm{i}, \mathrm{j}}^{0}$, то в качестве элемента $\mathrm{x}^{0}{ }_{\mathrm{i}, \mathrm{j}}$ таблицы $\mathrm{X}^{0}{ }_{131,6}$ берется значение средневзвешенного от этих нескольких значений. Такое усреднение необходимо из-за наличия нескольких однородных ГЦБ разных выпусков со сходными параметрами из 14 приведенных в БД. Объектом является набор доходностей однородных ГЦБ. Каждая строка такой таблицы относится к определенной дате проведения аукциона. Объект характеризуется значениями его 6 показателей. Временной ряд доходностей с заданным сроком до даты погашения называется изотермным рядом. Изотермному ряду из ј-го интервала поставим в соответствие гипотетический финансовый инструмент №j (ФИ №j) с доходностью указанного вида. Любое значение доходности ГЦБ, принадлежащее по значению показателя «количество дней до даты погашения» к ј-ому интервалу участвует в процессе формирования изотермного ряда из ј-го столбца матрицы $\mathrm{X}^{0}{ }_{131,6}$ Оценка степени риска ФИ №j является оценкой риска изменения значения доходности указанной ГЦБ. Элементы матрицы $\mathrm{X}_{131,6}^{\circ}$ определены на момент времени t, в другие моменты времени ее крайний правый столбец может исчезнуть. Например, если временная структура состоит из 5 интевалов, где 5-ый интервал «дата погашения» ГЦБ соответствует интервалу [181 дней - 360 дней]» и в течение 1 года не было ни одной эмиссии валютного ГЦБ, то через 360 (365 календарных) банковских дней «временная структура процентных ставок» ГЦБ сокращается (время жизни ГЦБ уменьшается) и будет состоять из 4-х интервалов.

На валютных аукционах ГЦБ РК, проходивших $\quad$ с 26.06 .99 г. $\quad$ по $29.12 .99 \quad$ г. проводились продажи ГЦБ типов ГКВО-3, Валютные ноты, СВГО-60, KASAKHSTAN-02, KASAKHSTAN-99. Число характеристик (параметров ГЦБ), как именных встроенных по умолчанию, так и появившихся после перерасчетов, равно 14: 3-даты, 5-числовые,4наименования, 2-порядковые номера). Для визуализации предпочтительности одних ГЦБ перед другими (в фиксированной временной структуре процентных ставок) применяют «кривые доходности». С течением времени «процентные доходы» (\%-ты от номинальной цены ГЦБ), получаемые от инвестиций в ГЦБ менялись и это явление привело к другому явлению - изменению «доходности к дате погашения». Эту зависимость от времени жизни ГЦБ (от временной структуры процентных ставок) представляют графически, и называется она «кривая доходности». Если кривая параллельна оси абсцисс, то это указывает на тот факт, что абсолютно для всех ГЦБ процентная ставка остается одинаковой.

\section{Таблица «объект-свойство».}

В базе данных для определения кривой доходности фиксированных 6 финансовых инструментов (ФИ) использовались только 4 показателя из 5: дата операции (продажи), «количество дней до даты погашения», вид сделки, «доходность к дате погашения» (в \%-ах), тип ГЦБ. Из БД формируем таблицу "объект свойство" $\mathrm{X}_{131,6}^{0}$, состоящую из 131 строк-

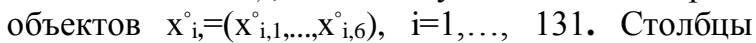
(изотермные ряды) образуют коррелированную систему свойств объектов. Предлагаемый нами многомерный анализ таких данных имеет другую временную структуру и выявляет отличное от полученных в [16-18]. Общим местом в этих работах является применение ПМ ГК [1,3]). Разнятся содержательные смыслы показателей (их значения располагаются по столбцам таблицы "объект-свойство" $\mathrm{X}_{131,6}^{0}$ ) Такое применение ПМ ГК не зависит от форм кривых доходности [510,12-13,16-18], могущих образовывать разные формы. Временной ряд из случайных значений $\mathrm{x}_{\mathrm{i}, \mathrm{j}}^{\circ}, \quad \mathrm{i}=1, \ldots, 131, \quad \mathrm{j}=1, \ldots, 6, \quad$ бескупонных доходностей из заданных (из ј-го интервала временной структуры, из ј-го узла кривой доходности) интервалов «времен жизни» ГЦБ) располагается в ј-ом столбце матрицы исходных данных $\mathrm{X}^{0}{ }_{131,6}$ и называется изотермным рядом [12]. Доходность ГЦБ к дате погашения есть внутренняя норма дохода, устанавливающая равенство между текущей стоимостью потока будущчих доходов и рыночной ценой ГЦБ к дате ее продажи на аукционе. Одним из влияющих на эту доходность факторов является длительность периода (время жизни), остающегося до погашения ГЦБ. Изменение цены ГЦБ происходит по 3 причинам [12-13]. У одних конкретных ГЦБ, привлекательных по своим параметрам, их значение «доходности до даты 
погашения» на графике «кривой доходности» переходит из (i+1)-го узла в i-ый узел, или интересные для покупателя по этому виду доходности ГЦБ «располагаются» в первых 2-х, или 3-х интервалах. Чем меньше номер интервала, тем выше доходность ГЦБ. Любое изменение временной структуры процентных ставок ГЦБ влияет на цену того или иного ГЦБ. Самой неопределенной является рыночная цена ГЦБ, зависящая от многих и разнородных рыночных и нерыночных факторов. Эти фактры порождают причины возникновения риска изменения доходностей. В [13] объясняется применение ПМ ГК[1] для решения задачи определения значений в узлах кривой доходности (погасительного профиля временной структуры процентных ставок). Дан алгоритм сведения (свертки) изотермных рядов в некоррелированные временные ряды значений доходностей в узлах кривой доходности.

Рассматриваемый в статье вид процентного риска связан с неопределенностью изменения значений «доходности к дате погашения» на кривой доходности конкретной ГЦБ при переходе «срока жизни» этой ГЦБ из ј-го интервала в (j-1)-ый интервал, $\mathrm{j}=2, \ldots, 6$. Неопределенность изменений значений процентных ставок абстрагируется в реализациях 6 зависимых 1-мерных теоретических случайных величин $\xi_{1}, \ldots, \xi_{6}$ с неизвестными законами распределения, поставленных в соответствие 6 интервалам нашей временной структуры. Статистические взаимосвязи значений процентных ставок из интервалов №j, j=1,.,6, временной структуры измеряяются значениями парных коэффициентов корреляции Пирсона между парами рассматриваемых ниже переменных. С учетом 3-х видов коэффициентов корреляций и дисперсий переменных ниже вычислены значения процентного риска изменения «доходности к дате погашения» для валютных ГЦБ Республики Казахстан. В расчетах приводим цифры в долларах, но цифры соответствуют и другим видам валют, ибо наши формулы применимы при любом линейном преобразовании элементов анализируемой таблицы.

\section{Неопределенность и риски изменения} доходностей.

Поставим в соответствие процентным ставкам (доходностям) $\mathrm{x}_{\mathrm{i} 1}^{0}, \ldots, \mathrm{x}_{\mathrm{i}}^{0}$ теоретические случайные величиины $\xi_{1}, \xi_{2}, \xi_{3}, \xi_{4} \xi_{5}, \xi_{6}$, а оценки стандартных отклонений их реализаций (изотермные ряды из элементов столбцов с номерами $1,2,3,4,5,6)$ используем в качестве измерителей степени волатильности (рисковости) изменения соответствующих доходностей ФИ. Квадрат значения стандартного отклонения - дисперсия, измеряет степень поиска изменения процентной ставки типа «доходность к дате погашения». Так как доходность ГЦБ - это результат как нестохастического процесса (приближение даты погашения происходит посуточно), так и случайных изменений процентных ставок (избыточных доходностей) из-за конъюнктурных изменений ситуации на рынке ГЦБ, то необходимо в данных из БД удалить нестохастическую составляющую. Полной доходности ГЦБ мы поставим в соответствие случайную величину $\xi_{\mathrm{j}}$, реализации которой считаем равными значениям $\mathrm{x}_{\mathrm{i}, \mathrm{j}}^{0}$ процентных ставок на валютных аукционах ГЦБ РК. Далее мы работаем не с полной доходностью, а с избыточной доходностью и определяем риск изменения доходности ГЦБ как дисперсию избыточной доходности ГЦБ. Кратко поясним это, опираясь на работу [13]. Избыточная доходность равна доходности ГЦБ с нулевым купоном минус (-) безрисковая доходность. Безрисковая доходность - это доходность данной облигации с нулевым купоном, меняющаяся только за счет приближения даты погашения. Отсюда следует, что мы должны начать с определения доходности ГЦБ и безрисковой доходности. Чтобы понять определение доходности, рассмотрим одномесячный период владения ГЦБ. Пусть в текущий момент времени $\mathrm{t}$ период владения ГЦБ до продажи еe на аукционе равен $\Delta \mathrm{t}$, а срок до погашения в $\mathrm{j}$-ом интервале равен $\mathrm{w}_{\mathrm{j}}, \mathrm{j}=1.2 .3 .4 .5 .6$. Тогда срок жизни ГЦБ в момент времени $\mathrm{t}$ равен $\Delta \mathrm{t}+\mathrm{w}_{\mathrm{j}}$. Рассмотрим инвестирование $1 \$$ в момент времени $\mathrm{t}$ в ГЦБ со сроком до погашения $\mathrm{t}+\Delta \mathrm{t}+\mathrm{w}_{\mathrm{j}}$ и продажу в момент времени $\mathrm{t}+\Delta \mathrm{t}$. Случайное изменение стоимости инвестиций за время $\Delta \mathrm{t}+\mathrm{w}_{\mathrm{j}}$ $\left(\Delta \mathrm{t} \geq 0, \mathrm{w}_{\mathrm{j}}>0\right)$ обладания ГЦБ и будет доходностью за этот период. Изменение доходности будет определяться как с приближением даты погашения (с изменением временной структуры ГЦБ, номер j интервала жизни для фиксированного вида ГЦБ перемещается влево на оси интервалов, что видно в Таблице 4, где стрелки, направлены справа налево), так и в связи с восприятием участниками рынка характеристик ГЦБ. Тем самым за время $\Delta \mathrm{t}+\mathrm{w}_{\mathrm{j}}$ жизни на бирже ГЦБ меняет свой дисконтный тип на рисковый тип.

Для иллюстрации одного из деталей этого процесса рассмотрим инвестирование в ГЦБ 1 в в момент времени $\mathrm{t}$ в дисконтный период (тип ГЦБ дисконтный) со сроком до даты погашения $\mathrm{t}+\Delta \mathrm{t}$. Доходность этой инвестиции будет абсолютно определенной (неслучайной, ибо ее значение вычислено и зафиксировано в базе данных KASE), и, следовательно, безрисковой. Тогда доходности ГЦБ рискового типа с датой 
погашения $\mathrm{t}+\Delta \mathrm{t}+\quad \mathrm{w}_{\mathrm{j}} \quad(\mathrm{j}=1, \ldots, 6)$ минус $\quad$ их безрисковые доходности равны избыточным доходностям ГЦБ рисковых типов. Мы рассматриваем денежные потоки этих избыточных доходностей ГЦБ, погашаемых в узлах $\mathrm{t}+\Delta \mathrm{t}+\mathrm{w}_{\mathrm{j}}$ (время жизни равно $\Delta \mathrm{t}+\mathrm{w}_{\mathrm{j}}$ ) временной структуры процентных ставок. Далее мы рассматриваем только избыточные доходности. При вычислении безрисковой доходности по формуле вычисления доходности ГЦБ с датой погашения $\mathrm{t}+\Delta \mathrm{t}+\mathrm{w}_{\mathrm{j}}$ любое случайное изменение стоимости ГЦБ (изменение цены мы не рассматриваем), из-за приближения даты погашения будет удалено. Для ГЦБ рискового типа имеем значения процентных ставок (годовых) по 6 интервалам $\Delta \mathrm{t}+\mathrm{w}_{\mathrm{j}}, \mathrm{j}=1, \ldots, 6$, временной структуры, которым мы для упрощения изложения, поставим в соответствие 6 финансовых инструментов (ФИ) фондового рынка РК с избыточными доходностями.

Возникающие в 6 узлах кривой доходности значения избыточных доходностей (коррели рованных доходностей), характеризуют зависимости ФИ друг с другом. Таким образом полученная избыточная доходность является чисто стохастическим элементом [13]. Так как число таких элементов в момент времени $\mathrm{t}$ $(\mathrm{t}=1, \ldots, 131)$ равно 6 , то образуется наша таблица $\mathrm{X}_{131,6 .}^{\circ}$ Эту таблицу рассматриваем как случайную многомерную выборку из генеральной совокупности с неизвестным законом распределения. Обоснование неизвестности законов распределения 1-мерных случайных переменных, имеющих разные оценки эмпирической функции плотности распределения. (их t-ые значения суть $\mathrm{x}_{\mathrm{t} 1}^{\circ}, \ldots, \mathrm{x}_{\mathrm{t} 6}^{\circ}$ ), $\mathrm{t}=1, \ldots, 131$, имеется в [19].

Мы не рассматриваем рыночные цены, а исследуем «доходности к дате погашения», для которых сроки погашения являются самыми важными влияющими факторами. Оценим значения теоретических ожидаемых доходностей (нам неизвестных). 6 временных рядов доходностей (изотермных рядов) за периоды владения для каждого узла временной структуры. Оценим риск изменения процентных ставок типа «доходность к дате погашения», присущий каждому ФИ, но неизвестный как продавцу, так и покупателю. Задача вычисления этих рисков усложняется тем, что рыночная доходность (случайная), формируется под влиянием макро- и микроэкономических факторов, поэтому цене ставится в соответствие случайная величина, имеющая неизвестную функцию распределения, а конкретные значения этой случайной величины принимаются в качестве измеренных значений рыночной доходности. Это происходит из-за того, что разные ГЦБ имеют разные ликвидности, сроки погашения, номинальные ставки дохода и другие особенности и характеристики.

Под рисковостью процентной ставки типа «доходность к дате погашения» мы понимаем отклонение действительного значения процентной ставки на аукционе от ожидаемой процентной ставки (в вероятностных терминах от математического ожидания генеральной совокупности процентных ставок). Оценкой математического ожидания (наиболее вероятного значения - служит выборочная средняя, вычисленная у нас по выборке из 131 значений процентных ставок, зафиксированных на аукционах, проходивших с 26.06 .1999 по 29.12.1999г. В 2-ом полугодии 1999 года проходили 131 аукционов на Казахстанской фондовой бирже (KASE) по видам ГЦБ, классифицированных по 6 рассматриваемым нами срокам до даты погашения. Степень рисковости изменения процентной ставки будем измерять степенью отклонения фактических значений процентной ставки ФИ на аукционах от ожидаемой процентной ставки ФИ. Из многих измерителей степени рисковости процентной ставки будем использовать один - квадрат выборочного стандартного отклонения $\mathrm{s}^{2}{ }_{\mathrm{j}}$ дисперсию, вычислен ную по той же выборке из 131 значений процентных ставок. Значение $\mathrm{s}_{\mathrm{j}}$, измеряет степень волатильности. Чем выше разброс значений процентных ставок, тем выше вероятность получения большего дохода владельцем ГЦБ, купившего ее на аукционе эмитентов. Для портфеля ГЦБ существует ожидаемый доход и ожидаемый риск (этотермины финансового анализа). В терминах количественных методов финансового анализа эта фраза формулируется в виде гипотезы: «для генеральной совокупности значений процентной ставки типа «доходность к дате погашения» существуют математическое ожидание доходности и дисперсии (меры риска изменения доходности». Предполагается существование теоретической функции распределения случайной величины, поставленной в соответствие с переменным «доходность». Предполагается также, что существует неизвестная нам функциональная связь между «доходностью» и «риском». Если значениям показателя «доходность» ГЦБ поставим в соответствие реализа ции теоретической случайной величины $\xi$, то теоретическая «доходность» ГЦБ есть линейная функция от математического ожидания случайной величины $\xi$, а теоретический «риск» есть среднеквадратичное отклонение от математического ожидания случайной величины $\xi$, т.е. является функцией от теоретической дисперсии случайной величины $\xi$, реализации которой равны доходностям ГЦБ, входящих в 
портфель инвестора. Вид функциональной связи между «доходностью» и «риском» портфеля из нескольких ГЦБ, будучи изображенной на диаграмме, имеет сложный вид. Особенностью рассматриваемой нами кривой является соответствие одному значению «доходности» многих значений «риска», и соответствие одному значению «риска» многих значений «доходности». Имеется много исследований анализа типов кривой доходности [5-9,14,16-18].

Возможная комбинация ФИ (ФИ №j состоит из ГЦБ, срок жизни которых принадлежит интервалу №j временной структуры), входящих в портфель, зависит от нескольких функций полезности доходности или другой характеристики для инвестора. Другое дело, что она - функция полезности, как правило, решает одну задачу. Но мы ниже решаем задачу сохранения суммы рисков при преобразовании доходностей 6 ФИ, дополнив ее задачей минимизации условной упущенной выгоды, например, в шкале измерения рисков: если вычисленный риск равен 70\%, то упущенная выгода равна $30 \%$. Решаемая задача перехода к независимым ФИ при 2 дополнительных критериях (смотрите ниже) вычисляет упущенную выгоду так, как показано в Таблице 3 в виде выявления и показа игнорируемых дилером значений рисков ФИ из НФИ №4, НФИ №5, НФИ №6.

Рассмотрим парные связи между доходностями 6 ФИ, меры тесноты связи между ними отражены в выборочной корреляционной матрице (Таблица 1). Решим задачу выделения комбинаций видов ФИ, образующих высокорисковые (по значениям дисперсий $\lambda_{1}, \lambda_{2}$ $\lambda_{3}$ ) новые ФИ - новые локальные портфели (НФИ
№1, НФИ №2, НФИ №3) «заметных» ФИ (по критерию 2), значимые риски которых мы вычисляем и фиксируем на момент времени $\mathrm{t}$. Величины указанных рисков зависят от степени коррелированности рассматриваемых ниже парных коэффициентов корреляций Пирсона $\mathrm{r}_{\mathrm{ij}}{ }_{\mathrm{ij}}=\operatorname{corr}^{2}\left(\mathrm{z}_{\mathrm{i}}, \mathrm{z}_{\mathrm{j}}\right), \quad \lambda_{\mathrm{j}}^{2}=\operatorname{corr}^{2}\left(\mathrm{y}_{\mathrm{j}}, \mathrm{y}_{\mathrm{j}}\right), \quad \mathrm{c}_{\mathrm{ij}}{ }_{\mathrm{ij}}=\operatorname{corr}^{2}\left(\mathrm{z}_{\mathrm{i}}, \mathrm{y}_{\mathrm{j}}\right)$, вычисляемых ниже при применении моделей из [1-3].

Из таблицы "объект-свойство" $\mathrm{X}^{0}{ }_{131,6}$ рассчитываем усредненные характеристики 6 ФИ такими, как они сложились под влиянием всех рыночных факторов. Если бы инвестор знал эти характеристики, то старался бы формировать свой портфель продаж ГЦБ, аналогичный портфелю эмитента по продаже ГЦБ, по структуре, по срокам владения валютных ГЦБ до их даты погашения и по другим характеристикам (их 14). Отметим, что портфель эмитента по продаже ГЦБ не обязательно является оптимальным портфелем (с минимальной дисперсией). Для коммерческих банков важно знать всё про портфель продаж валютных ГЦБ, чтобы выгодно покупать некоторые валютные ГЦБ, а потом продать их с другой выгодой.

Оценки ожидаемых значений процентных ставок 6 ФИ оказались примерно одинаковыми ( $\approx 10 \%$ годовых): $11.0496 \%, 10.2170 \%, 9.7309 \%$, $10.9450 \%, 10.7122 \%, 11.7651 \%$. Но оценки $\mathrm{s}_{\mathrm{j}}$

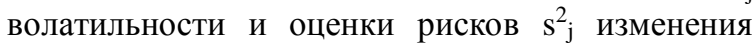
процентных ставок для 6 наших ФИ (интервалов) оказались разными (Рисунок 1): $\mathrm{s}_{1}=5.7072 \%, \mathrm{~s}_{2}=4.1082 \%, \mathrm{~s}_{3}=2.4496 \%$, $\mathrm{s}_{4}=1.3742 \%, \mathrm{~s}_{5}=0.6732 \%, \mathrm{~s}_{6}=1.4664 \%$, $\mathrm{s}^{2}{ }_{1}=32.572 \%, \mathrm{~s}_{2}{ }_{2}=16.8773 \%, \mathrm{~s}^{2}{ }_{3}=6.00054 \%$, $\mathrm{s}^{2}{ }_{4}=1.8884 \%, \mathrm{~s}_{5}^{2}=0.4532 \%, \mathrm{~s}_{6}^{2}=2.15 \%$. 


\begin{tabular}{|c|c|c|c|c|c|c|}
\hline Impact Factor: & $\begin{array}{l}\text { ISRA (India) } \\
\text { ISI (Dubai, UAE } \\
\text { GIF (Australia) } \\
\text { JIF }\end{array}$ & $\begin{array}{l}=1.344 \\
=0.829 \\
=0.564 \\
=1.500\end{array}$ & $\begin{array}{l}\text { SIS (USA) } \\
\text { PИНЦ (Russia) } \\
\text { ESJI (KZ) } \\
\text { SJIF (Morocco) }\end{array}$ & $\begin{array}{l}=0.912 \\
=0.207 \\
=\mathbf{3 . 8 6 0} \\
=\mathbf{2 . 0 3 1}\end{array}$ & $\begin{array}{l}\text { ICV (Poland) } \\
\text { PIF (India) } \\
\text { IBI (India) }\end{array}$ & $\begin{array}{l}=6.630 \\
=1.940 \\
=4.260\end{array}$ \\
\hline
\end{tabular}

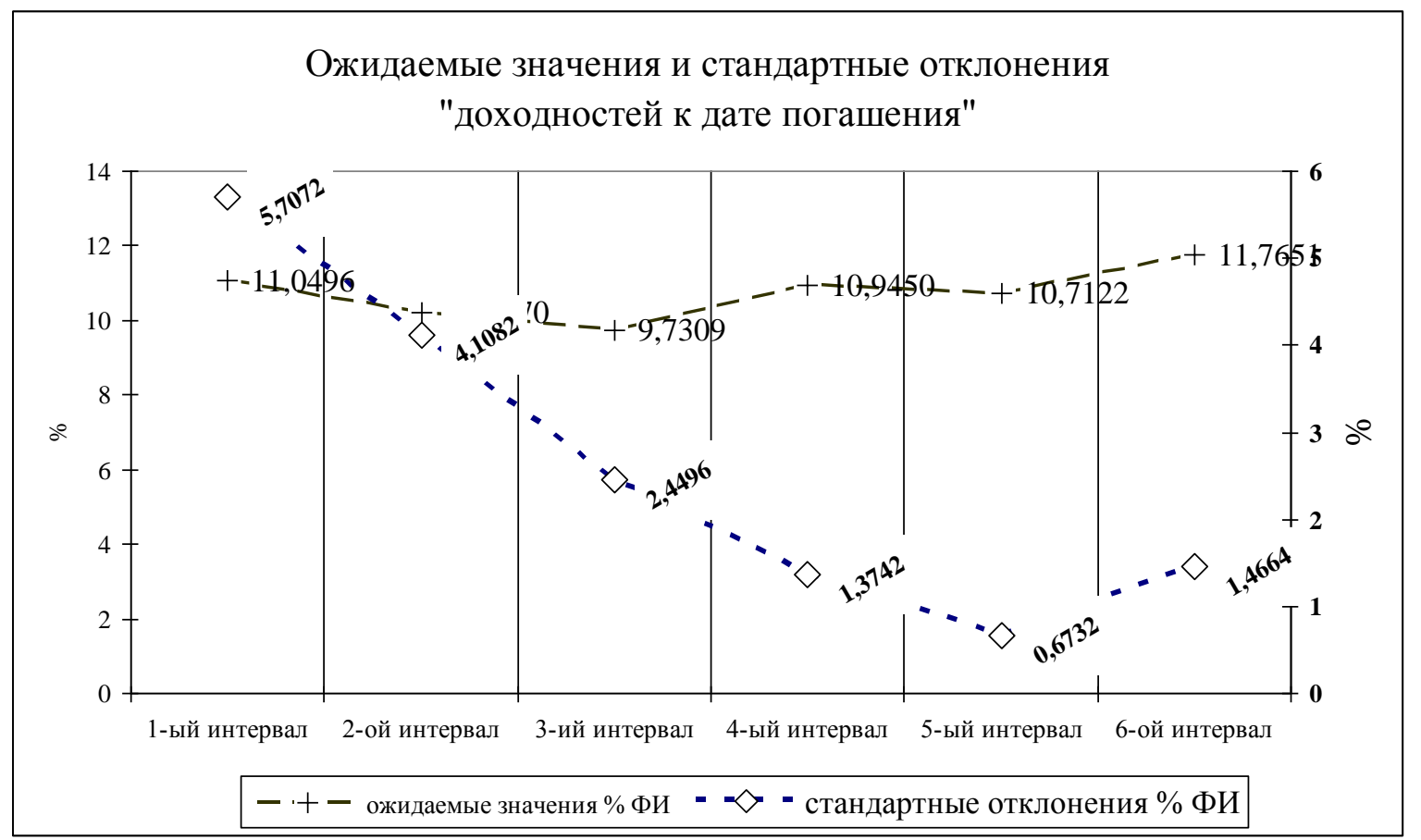

Рисунок 1 Динамики ожидаемых значений процентных ставок и степеней волатильности 6 ФИ

Параметр стандартного отклонения $\sigma$ совокупности, $s=+\sqrt{ } s^{2}$ - выборочное стандартное отклонение (оценка $\sigma$ ) характеризует степень изменчивости (волатильности) х-переменной $\left(\mathrm{x}=\mathrm{x}^{\circ}-\mathrm{x}_{\mathrm{cp}}\right), \quad$ а значение $\mathrm{z}$-переменной равно $\mathrm{z}=\left(\mathrm{x}^{\circ}-\mathrm{x}_{\mathrm{cp}}\right) / \mathrm{s} . \quad$ Формула $\mathrm{x}^{\circ}=\mathrm{x}_{\mathrm{cp}}+\mathrm{zs} \quad$ показывает структуру разложения измеренного значения $\mathrm{x}^{\circ}$ на слагаемые. Первое слагаемое $\left(\mathrm{x}_{\mathrm{cp}}\right)$ называется ожидаемым значениием, оно является главной частью значения $\mathrm{x}^{\circ}$ реального показателя ФИ и имеет единицу измерения: $\mathrm{x}_{\mathrm{cp}}=10 \%$. Второе слагаемое (zs) показывает число $\mathrm{z}=\left(\mathrm{x}^{0}-\mathrm{x}_{\mathrm{cp}}\right) / \mathrm{s}$ отклонений (стандартных) в отклонении исходного значения $\mathrm{x}_{\mathrm{ij}}^{\circ}$ от значения выборочного среднего: $x_{\mathrm{ij}}=\left(\mathrm{x}_{\mathrm{ij}}^{\circ} \mathrm{x}_{\mathrm{j}}^{\mathrm{cp}}\right), z_{\mathrm{ij}}=x_{\mathrm{ij}} / \mathrm{s}_{\mathrm{j}}$, где $\mathrm{x}_{\mathrm{ij}}=\left(\mathrm{x}^{\circ}-\mathrm{x}_{\mathrm{j}}^{\mathrm{cp}}\right)=z_{\mathrm{ij}} \mathrm{s}_{\mathrm{j}}$. Если $\mathrm{x}_{\mathrm{ij}}^{\circ}=12 \%, \mathrm{x}_{\mathrm{j}}^{\mathrm{cp}}=8 \%, \mathrm{sj}_{\mathrm{j}}=4 \%$, то $\mathrm{x}_{\mathrm{ij}}^{\circ}$ отделен от своего ожидаемого значения $\mathrm{x}_{\mathrm{j}}^{\mathrm{cp}}=8 \%$ расстоянием в 1 стандартное отклонение: $z_{\mathrm{ij}}=(12-8) / 4=1$.

Убывающая динамика значений волатильности (Рисунок 1) по мере увеличения сроков жизни правильно отражает тенденцию снижения рисков изменения процентных ставок выбранного нами типа. Но их значения не учитывают различные степени взаимосвязей между изотермными рядами. Из-за наличия взаимозависимости друг от друга величин доходностей из разных интервалов временной структуры приведенные значения $\mathrm{s}_{1}, \mathrm{~s}_{2}, \mathrm{~s}_{3}, \mathrm{~s}_{4}, \mathrm{~s}_{5}, \mathrm{~s}_{6}$ степени волатильности не пригодны для оценки значений риска изменения доходностей наших ФИ. ГЦБ различаются также условиями досрочного погашения, условиями обмена на другую ЦБ, разными степенями риска обладают цены продаж коротких, краткосрочных, долгосрочных ГЦБ. Эти и другие свойства влияют на оценки рисков изменения доходностей в ј-ых интервалах (j-ых ФИ) временной структуры. Приведенные выше значения степени рисковости доходностей 6 ФИ разные, но они не учитывают значений степени коррелированности между парами ФИ, приведенных в матрице парных коэффициентов корреляций (таблица 1). В формулах [3], определяющих значения рисков должен присутствовать ненулевой коэффициент корреляции $\mathrm{r}_{\mathrm{ij}}$ зависящей от элементов $\mathrm{Z}_{\mathrm{ki}}, \mathrm{Z}_{\mathrm{kj}}$ стандартизованной выборки $Z_{131,6}=\left\{z_{i j}\right\}$, каждый элемент которой получен преобразованием элемента $\mathrm{x}_{\mathrm{ij}}{ }^{0}$ в безразмерный элемент матрицы $\mathrm{Z}_{131,6}=\left\{\mathrm{Z}_{\mathrm{ij}}\right\}, \mathrm{i}=1, \ldots, 131, \mathrm{j}=1, \ldots, 6$, где $\mathrm{z}_{\mathrm{ij}}=\left(\mathrm{x}_{\mathrm{ij}}{ }^{\circ}-\mathrm{x}_{\mathrm{j}}{ }^{\mathrm{cp}}\right) / \mathrm{s} \mathrm{j}$, $\mathrm{x}_{\mathrm{j}}^{\mathrm{c}} \mathrm{p}=\left(\mathrm{x}_{1, \mathrm{j}}^{\circ}+\ldots+\mathrm{x}_{131, \mathrm{j}}^{\circ}\right) / 131, \quad \mathrm{~s}_{\mathrm{j}}{ }^{2}=\left(\mathrm{x}_{1 \mathrm{j}}{ }^{2}+\ldots+\mathrm{x}_{131, \mathrm{j}}{ }^{2}\right) / 131$, $\mathrm{x}_{\mathrm{ij}}=\mathrm{x}_{\mathrm{ij}}{ }^{\circ}-\mathrm{x}_{\mathrm{j}}^{\mathrm{cp}}, \quad \mathrm{k}=1, \ldots, 131, \quad \mathrm{i}=1, \ldots, 131, \mathrm{j}=1, \ldots, 6$. Здесь $Z_{131,6}$ - входной элемент решаемой ниже П3 АГК $[1,3]: \mathrm{Z}_{131,6}=>\left(\mathrm{R}_{66}, \mathrm{C}_{66}, \Lambda_{66}, \mathrm{Y}_{131,6}\right)$.

Степень рисковости портфеля из доходностей 6 ФИ вычисляем с учетом значений степеней рисковости доходностей 6 ФИ и значений выборочных коэффициентов корреляций между парами z-переменных, число которых равно (6-1)*6/2=15. Но нам нужны ФИ с достаточно высокими значениями рисков изменения значений процентных ставок типа «доходность к дате погашения», а им соответствуют только несколько коэффициентов корреляции, удовлетворяющих Критерию 1 (см. ниже). Эти коэффициенты корреляции 
отличаются от других реально воспринимаемых величин, характеризующих ФИ.

\section{Формулы вычисления значений} процентных рисков изменения доходности к дате погашения для высокорисконых ГЦБ. Практически важные характеристики портфеля ГЦБ не ограничиваются только этими параметрами. Наиболее важными являются риски изменения процентных ставок типа «доходность к дате погашения». Риск изменения рассматриваемого нами типа процентной ставки для портфеля независимых ФИ измеряется суммой дисперсий соответствующих некоррелированных нормированных значений процентных ставок временных рядов. Для определения риска портфеля зависимых ФИ необходимо вычислять значения парных коэффициентов корреляции Пирсона, как показано ниже, двух видов: $\mathrm{r}_{\mathrm{ij}}=\operatorname{corr}\left(\mathrm{z}_{\mathrm{i}}, \mathrm{z}_{\mathrm{j}}\right)$, $\mathrm{c}_{\mathrm{ij}}=\operatorname{corr}\left(\mathrm{z}_{\mathrm{i}}, \mathrm{y}_{\mathrm{j}}\right)$, и одну дисперсию $\lambda_{\mathrm{j}}=\operatorname{corr}\left(\mathrm{y}_{\mathrm{j}}, \mathrm{y}_{\mathrm{j}}\right)$. Интересно то, что формула риска портфеля зависимых ФИ, которым соответствуют zпеременные, не содержит формулу дисперсии zпеременной: $\operatorname{corr}\left(\mathrm{z}_{\mathrm{j}}, \mathrm{z}_{\mathrm{j}}\right)=1, \quad \mathrm{j}=1, \ldots, 6 . \quad$ Формулы вычисления рисков портфелей ФИ с заметно высокими рисками будут обоснованы и вычислены ниже для реальных данных.

Чтобы обеспечить высокую доходность, «зависящей от воспринимаемого инвестором риска, и, следовательно, не зависящей от уровня цены актива» [12], инвестор должен иметь портфель с высоким риском: покупать ГЦБ, входящие в высокорисковые ФИ, формировать новые независимые высокорисковые портфели из видов ГЦБ с «выгодными» параметрами. Разные ГЦБ имеют разные значения «доходности к дате погашения» с разными степенями случайности и волатильности, а рисковые ГЦБ - положительные ожидаемые доходы, измеряемые, как выше показано, средними арифметическими $\mathrm{x}^{\mathrm{cp}} 1, \ldots$, $\mathrm{x}^{\mathrm{cp}}$. Независимость двух НФИ №i и №j реализуется посредством моделирования двух безразмерных временных рядов, нормированных и некоррелированных значений у-переменных №i и №j таких, что $\mathbf{y}_{\mathbf{i}}=\left(\mathrm{y}_{1 \mathrm{i}}, \ldots, \mathrm{y}_{131, \mathrm{i}}\right)^{\mathrm{T}}, \mathbf{y}_{\mathbf{j}}=\left(\mathrm{y}_{1 \mathrm{j}}, \ldots, \mathrm{y}_{131, \mathrm{j}}\right)^{\mathrm{T}}$, $(1 / 131) \mathbf{y}_{i}^{\mathrm{T}} \mathbf{y}_{\mathbf{j}}=0, \quad(1 / 131) \mathbf{y}_{\mathbf{i}}^{\mathrm{T}} \mathbf{y}_{\mathbf{i}}=0, \quad(1 / 131) \mathbf{y}_{\mathbf{j}}^{\mathrm{T}} \mathbf{y}_{\mathbf{j}}=\lambda_{\mathrm{j}}$, $\mathrm{i}, \mathrm{j}=1, \ldots, 6$. При этом сумма дисперсий 6 новых упеременных должна равняться сумме дисперсий зависимых ФИ №i и №j, реализованных посредством вычисления двух временных рядов безразмерных, нормированных и коррелирован ных значений z-переменных №i и №j таких, что $\mathbf{Z}_{\mathbf{i}}=\left(\mathrm{z}_{1 \mathrm{i}}, \ldots, \mathrm{Z}_{131, \mathrm{i}}\right) \quad{ }^{\mathrm{T}}, \mathbf{Z}_{\mathbf{j}}=\left(\mathrm{z}_{1 \mathrm{j}}, \ldots, \mathrm{Z}_{131, \mathrm{j}}\right)^{\mathrm{T}}, \quad(1 / 131) \mathrm{z}_{\mathrm{i}}^{\mathrm{T}} \mathbf{Z}_{\mathrm{j}}=\mathrm{r}_{\mathrm{i}, \mathrm{j}}$, (1/131) $\mathbf{z}_{\mathbf{i}}^{\mathrm{T}} \mathbf{z}_{\mathbf{i}}=1, \quad(1 / 131) \mathbf{z}_{\mathbf{j}}^{\mathrm{T}} \mathbf{z}_{\mathbf{j}}=1, \quad \mathrm{i}, \mathrm{j}=1, \ldots, 6$. Это условие сохранения неизменной сумму дисперсий (сумму рисков ФИ) имеет вид: $1+1+1+1+1+1=\lambda_{1}+\ldots+\lambda_{6}=6$. Это следует из того, что диагональные элементы выборочной корреляционной матрицы $\mathrm{R}_{66}=(1 / 131) \mathrm{Z}^{\mathrm{T}}{ }_{131,6} \mathrm{Z}_{131,6}$ равны $1: \operatorname{diag}\left(\mathrm{R}_{66}\right)=(1, \ldots, 1)$, а сумма их равна 6 : $\operatorname{tr}\left(R_{66}\right)=1+1+1+1+1+1=\operatorname{tr}\left(\Lambda_{66}\right)=\lambda_{1}+\ldots+\lambda_{6}=6$.

Как преобразовать z-переменные в переменные? В работах [5-10,13,16-18] применили успешно ортонормированную матрицу собственных векторов. Мы применяем матрицу $\mathrm{C}_{66}$ собственных векторов корреляционной матрицы $\mathrm{R}_{66}$ такую, что выполняются условия нормированности и ортогональности: $\mathrm{C}_{66}^{\mathrm{T}} \mathrm{C}_{66}=\mathrm{I}_{66}, \quad \mathrm{C}_{66} \mathrm{C}_{66}^{\mathrm{T}}=\mathrm{I}_{66}$. Она вычисляется по известной кореляционной матрице $\quad \mathrm{R}_{66}=(1 / 131) \mathrm{Z}^{\mathrm{T}}{ }_{131,6} \mathrm{Z}_{131,6}$ при решении прямой спектральной задачи (ПС3,[3]) $\mathrm{R}_{66}=>\left(\mathrm{C}_{66}, \Lambda_{66}\right)$. ПС3 - это прямая задача диагонализации известной выборочной корреляционной матрицы $\mathrm{R}_{66}$, решаемая для симметрической матрицы $\mathrm{R}_{66}=\mathrm{R}^{\mathrm{T}}{ }_{66}$, в результате решения которой вычисляются 2 матрицы: ортогональная матрица $\mathrm{C}_{66}$ собственных векторов $\mathbf{c}_{\mathbf{j}}=\left(\mathrm{c}_{1 \mathrm{j}}, \mathrm{c}_{2 \mathrm{j}} \ldots \mathrm{c}_{6 \mathrm{j}}\right)^{\mathrm{T}}$, расположенных по её столбцам: $\mathrm{C}_{66}=\left[\mathbf{c}_{1}\left|\mathbf{c}_{2}\right| \ldots \mid \mathbf{c}_{6}\right]$ и согласованная со спектром $\Lambda_{66}=\operatorname{diag}\left(\lambda_{1}, \ldots \lambda_{6}\right) \quad$ таким образом, что выполняются соотношения

$$
\begin{gathered}
\mathrm{R}_{66} \mathrm{C}_{66}=\mathrm{C}_{66} \Lambda_{66}, \mathrm{C}_{66}^{\mathrm{T}} \mathrm{C}_{66}=\mathrm{C}_{66} \mathrm{C}_{66}^{\mathrm{T}}=\mathrm{I}_{66}, \\
\operatorname{diag}\left(\mathrm{R}_{66}\right)=(1, \ldots, 1), \\
\operatorname{tr}\left(\mathrm{R}_{66}\right)=1+1+\ldots+1=\operatorname{tr}\left(\Lambda_{66}\right)=\lambda_{1}+\ldots+\lambda_{6}=6, \\
\lambda_{1} \geq \ldots \geq \lambda_{6} \geq 0 .
\end{gathered}
$$

Решениями ПСЗ являются 2 матрицы $\mathrm{C}_{66}$, $\Lambda_{66}$, а ортогональное преобразование - матрица $\mathrm{C}_{66}$, примененное к матрице $\mathrm{Z}$-переменных $\mathrm{Z}_{131,6}$ : $\mathrm{Y}_{131,6}=\mathrm{Z}_{131,6} \mathrm{C}_{66}$, не изменяет сумму дисперсий $\mathrm{Z}$ переменных: $\left.\quad 6=\operatorname{tr}\left(\mathrm{R}_{66}\right)=\operatorname{tr}\left[(1 / 131) \mathrm{Z}^{\mathrm{T}}{ }_{131,6} \mathrm{Z}_{16,4}\right)\right]=$ $=\operatorname{tr}\left[\left((1 / 131) \mathrm{C}_{66} \mathrm{Y}^{\mathrm{T}}{ }_{131,6} \mathrm{Y}_{131,6} \mathrm{C}_{66}^{\mathrm{T}}\right]=\operatorname{tr}\left(\mathrm{C}_{66} \Lambda_{66} \mathrm{C}^{\mathrm{T}}{ }_{66}\right]=\right.$ $\operatorname{tr}\left(\Lambda_{66} \mathrm{C}_{66}^{\mathrm{T}} \mathrm{C}_{66}\right]=\operatorname{tr}\left[\Lambda_{66} \mathrm{I}_{66}\right]=\operatorname{tr}\left(\Lambda_{66}\right)=\lambda_{1}+\ldots+\lambda_{6}=6$.

Выборка $\mathrm{Y}_{131,6}$ называется единственным решением вышеупомянутой ПЗ АГК. А выборка $Z_{131,6}$ называется ассоциированным решением П3 АГК[3]. Ортогональное преобразование $\mathrm{C}_{66}$, примененное к $Z_{131,6}$, не изменяет сумму дисперсий $\mathrm{z}$-переменных: $\mathrm{R}_{66}=(1 / 131) \mathrm{Z}^{\mathrm{T}}{ }_{131,6} \mathrm{Z}_{16,4}$. Пояснения насчет того почему переменные случайны в матрицах $\mathrm{Z}_{131,6}, \mathrm{Y}_{131,6}$ и почему случайны матрицы $\mathrm{R}_{66}, \mathrm{C}_{66}, \Lambda_{66}$, почему неизвестны их законы распределения вероятностей изложены в [3,19]. Единственное решение $\mathrm{Y}_{131,6}$ ПЗ АГК является одним бесконечного множества решений обратной задачи анализа главных компонент (О3 АГК) [3, стр.70-79] согласно теореме 2.3 [3,стр.109-111], $[20,21]$. Так как $\mathrm{Y}_{131,6}$ является решением (одним из бесконечного множества) ПЗ АГК, то согласно ОМ ГК [3], где известен входной параметр $\Lambda_{66}$, выполняется равенство $\Lambda_{66}=(1 / \mathrm{m}) \mathrm{Y}^{\mathrm{T}}{ }_{131,6} \mathrm{Y}_{131,6}$ и, тем самым, решена обратная спектральная задача (OC3): $\Lambda_{66}=>\left(\mathrm{C}^{(\ell)}{ }_{66}, \quad \mathrm{R}^{(\ell)}{ }_{66}\right), \quad \ell=1, \ldots, \infty$. Далее мы можем вычислить (C,Y)-выборки $\mathrm{Z}^{(\ell)}{ }_{131,6}=\mathrm{Y}_{131,6} \mathrm{C}^{(\ell) \mathrm{T}}, \ell=1, \ldots, \infty$, адекватные одной нашей реальной выборке $Z_{131,6}$. Выборки 
$\begin{array}{lrr}Z^{(\ell)}{ }_{131,6}=Y_{131,6} \quad \mathrm{C}^{(\ell) \mathrm{T}} & \text { имеют } & \text { выборочные } \\ \text { корреляционные } & & \text { матрицы } \\ \mathrm{R}^{(\ell)}{ }_{66}=(1 / \mathrm{m}) \mathrm{Z}^{(\ell) \mathrm{T}}{ }_{131,6} \mathrm{Z}^{(\ell)}{ }_{131,6 .} & \text { Как } & \text { показали }\end{array}$ $\begin{array}{ll}\mathrm{R}_{66}=(1 / \mathrm{m}) \mathrm{Z}_{131,6} \mathrm{Z}_{131,6 .} & \text { Как показали } \\ \text { вычислительные эксперименты с применением }\end{array}$ программы из ППП «Спектр» [22] пары индексов $(1,2),(4,5),(5,6)$ высоких коэффициентов корреляции из матрицы $\mathrm{R}_{66}$ удовлетворяющих Критерию 1, сохранили это качество и в большинстве из 1000 матриц $\mathrm{R}^{(\ell)} 66, \ell=1, \ldots, 1000$. Эти эксперименты проводились для нашего уже известного спектра $\quad \Lambda_{66}=(1 / \mathrm{m}) \quad \mathrm{Y}_{131,6}^{\mathrm{T}} \mathrm{Y}_{131,6}$ Решалась ОС3 $\Lambda_{66}=>\left(\mathrm{C}^{(\ell)}{ }_{66}, \mathrm{R}^{(\ell)}{ }_{66}\right), \ell=1, \ldots, 1000$, реализующая алгоритм Чалмерса (Chalmers C.P. [23]). Соответствующие решению $\mathrm{Y}_{131,6}$ ассоциированные решения $Z^{(\ell)}{ }_{131,6}=Y_{131,6} \mathrm{C}^{(\ell) \mathrm{T}}{ }_{66}$, $\left(\mathrm{C}^{(\ell)}, \Lambda, \mathrm{Y}\right)$-выборки $\mathrm{Z}^{(\ell)}{ }_{131,6}$, генерировались в OM АГК $[3,22]: \quad \Lambda_{66}=>\left(C^{(\ell)}{ }_{66}, \quad R_{66}{ }^{(\ell)}, Y_{131,6}, Z^{(\ell)}{ }_{131,6}\right)$, $\ell=1, \ldots, 1000$, модельно адекватны нашей выборке $\mathrm{Z}_{131,6}$ как $(\Lambda, \mathrm{Y})$-выборки. $(\mathrm{C}, \Lambda, \mathrm{Y})$-выборки [25] и $(\mathrm{C}, \Lambda)$-выборки [19] мы не рассматриваем, ибо нас интересуют вариабельности выделенных выше элементов $\mathrm{r}_{12}=0.6654, \quad \mathrm{r}_{45}=0.6221, \quad \mathrm{r}_{56}=0.4980$, расположенных в тех же строках и столбцах матриц $\mathrm{R}^{(\ell)}{ }_{66}, \ell=1, \ldots, 1000$, где неравенство вида $\mathrm{r}^{(\ell)}{ }_{56} \geq 0.5$ выполнялось при многих значениях номера $\ell$. Из теоремы 2.3 [3,20,21] следует, что одни и те же содержательные выводы получаются не только из анализа одной рассматриваемой здесь реальной $(\mathrm{C}, \Lambda)$-выборки, но из анализов других модельных $\left(\mathrm{C}^{(\ell)}, \Lambda\right)$ выборок, являющихся решениями ОЗ АГК [3]. ПЗ АГК решена в [1], О3 АГК решена в [2,3], а обратная модель главных компонент (ОМ ГК) и ее применения описаны в [3]. ПМ ГК является неотъемлемой частью ОМ ГК, но единственное решение ПЗ АГК содержится среди бесконечного множества решений О3 АГК, из этих решений, т.е.(C, $\Lambda)$-выборок) О3 АГК после анализа извлекаются одинаковые «цифровые» знания, приведенные ниже.

Мы используем дисперсию в качестве меры риска. Будем вычислять дисперсии 6 независимых у-переменных и, используя значения долей $\mathrm{Z}$-переменных в каждой выделенной (по критерию Кайзера-Дикмана [25]) у-переменной, вычислим доли дисперсий выделенных по Критерию 2 z-переменных. Критерий Кайзера-Дикмана [25] формулируется так: Выделять только у-переменные с дисперсиями, большими 1.

Квадратная корреляционная матрица $\mathrm{R}_{66^{-}}$ симметрическая: $\quad \mathrm{r}_{\mathrm{ij}}=\mathrm{r}_{\mathrm{ji}}$. Имеет достаточно простую структуру (Таблица 1), что говорит о специифичности развития фондового рынка валютных ГЦБ РК. Здесь рассмотрены коэффициенты парной корреляции, вычисленные по реальным данным за анализируемый период. В другие периоды времени они будут другие, но зависимость значений доминирующих $\ell$ (из всех
6) собственных чисел $\Lambda_{66}=\operatorname{diag}\left(\lambda_{1}, \ldots \lambda_{6}\right)$ от небольшого числа доминирующих элементов корреляционной матрицы $\mathrm{R}_{66}=(1 / 131) \mathrm{Z}_{131,6}^{\mathrm{T}} \mathrm{Z}_{131,6}$ выражена всегда. Можно было бы ограничиться только анализом коэффициентов корреляции доходностей ФИ, но это был бы плохой анализ, не выявляющий (не учитывающий рыночных факторов) скрытых закономерностей конкретного рынка валютных ГЦБ за анализируемый период времени.

Отсутствие ФИ №3 при анализе корреляционной матрицы вынуждает нас, как показано ниже, проводить более углубленный поиск коэффициентов корреляции.

\section{чисел. \\ Финансовая интерпретация собственных}

Вычисленные по известной корреляционной матрице числа $\lambda_{\mathrm{j}}, \mathrm{j}=1, \ldots, 6$, важны тем, что зависят от коэффициентов корреляции, а значение наибольшего элемента $\lambda_{1}$ тем больше, чем больше в наддиагональной части матрицы $\mathrm{R}_{66}$ число коэффициентов корреляции с доминирующими абсолютными значениями. Геометриическая интерпретация этого факта наглядно видна при $\mathrm{n}=2$. Если известна симметричная матрица $\mathrm{R}_{22}$, то по известной теории решается некоторая оптимизационная задача, которая сводится к решению уравнения относительно $\lambda: \operatorname{det}(\mathrm{R}-\lambda \mathrm{I})=0$, где det означает определитель матрицы $\left(\mathrm{R}_{22}-\lambda \mathrm{I}_{22}\right)$. $\mathrm{B}$ прямой спектральной задаче (ПС3) диагонализации матрицы $\mathrm{R}_{22}$ (так называется в [3] задача вычисления элементов спектра $\Lambda_{22}$ для известной матрицы $\mathrm{R}_{22}$ ) решения $\lambda_{1} \lambda_{2}$ находятся из условия равенства определителя нулю: $\operatorname{det}\left(\mathrm{R}_{22}-\lambda \mathrm{I}_{22}\right)=0$. Данное равенство преобразутся в уравнение вида $(1-\lambda)(1-\lambda)-\mathrm{r}_{12}\left(\mathrm{r}_{21}\right)=0$. Решениями являются 2 значения для $\lambda$. Раскрывая скобки и группируя члены, получим квадратное уравнение $\lambda^{2}-2 \lambda+\left(1-r^{2}{ }_{12}\right)=0$. Собственные числа $\lambda_{1}, \lambda_{2}$ явно выражаются через коэффициенты корреляции $\mathrm{r}_{21}=\mathrm{r}_{12}: \lambda_{1}=1+\mathrm{r}_{12}, \lambda_{2}=1-\mathrm{r}_{12}$, при этом $\lambda_{1}+\lambda_{2}=2$. Если $\mathrm{r}_{12}=1$, то $\lambda_{1}=2$, если $\mathrm{r}_{12}=0$, то $\lambda_{1}=1, \lambda_{2}=1$. Система из 2 уравнений вида $\mathrm{R}_{22} \mathbf{c}_{\mathrm{j}}=\lambda \mathbf{c}_{\mathbf{j}}$ при $\lambda_{1}=1+\mathrm{r}_{12}, \lambda_{2}=1-\mathrm{r}_{12}$, имеет собственные векторы $\mathbf{c}_{\mathbf{j}}=\left(\mathrm{c}_{1 \mathrm{j}}, \mathrm{c}_{2 \mathrm{j}}\right)^{\mathrm{T}}, \mathrm{j}=1,2$, такие, что $\mathbf{c}_{\mathbf{j}} \mathrm{T}_{\mathbf{j}} \mathbf{j}=1, \mathbf{c}_{.1}{ }^{\mathrm{T}} \mathbf{c}_{\mathbf{2}}=0$, $\mathrm{c}_{1}=(\cos \alpha,-\sin \alpha)^{\mathrm{T}}, \mathrm{c} .2=(\sin \alpha, \cos \alpha)^{\mathrm{T}}$ с координатами $(\cos \alpha,-\sin \alpha),(\sin \alpha, \cos \alpha)$. Единичные векторы нормированы: c.j ${ }^{\mathrm{T}} \mathrm{c} . \mathrm{j}=\cos ^{2} \alpha+\sin ^{2} \alpha=1, \quad$ c. ${ }^{\mathrm{T}} \mathrm{c} .2=\sin ^{2} \alpha+$ $+\cos ^{2} \alpha=1$, и ортогональны (взаимно перпендикулярны):

$$
\text { c. }{ }^{\mathrm{T}} \mathrm{c} .2=\cos (\alpha) \sin (\alpha)-\sin (\alpha) \cos (\alpha)=0 \text {. }
$$

Здесь $\alpha$ - угол, на который приподнята большая полуось эллипса (с длинами полуосей $\lambda_{1}>\lambda_{2}$ ) над 1-ой осью. Эту интерпретацию компонент собственных чисел и собственных векторов мы используем ниже при описании случайных ортогональных преобразований и при моделировании $\ell$-отклонений в $\mathrm{z}$-переменных 
$[3,22,23]$. Гиперэллипсоид при $\mathrm{n}>2$ важен тем, что внутри него находятся все 6-мерные точки с координатами $\left(\mathrm{z}_{\mathrm{k} 1}, \ldots, \mathrm{Z}_{\mathrm{k} 6}\right), \mathrm{\kappa}=1, \ldots, 131$. В общем случае $\mathrm{n}>2$ гиперэллипсоид [3,23] расположен наклонно по отношению к осям своей коорди натной системы. Финансовая интерпретация столбцов матрицы $Z_{131,6}$ - нормированные изотермные ряды.

Таблица 1

Матрица коэффициентов корреляции $\mathbf{R}_{6,6}$

\begin{tabular}{|l|c|c|c|c|c|c|}
\hline COLUMN & 1 & $\mathbf{2}$ & 3 & 4 & $\mathbf{5}$ & $\mathbf{6}$ \\
\hline ROW 1 & 1.000 & $\mathbf{0 . 6 6 5}$ & 0.298 & 0.0711 & 0.0552 & 0.1607 \\
\hline ROW 2 & 0.665 & 1.000 & 0.117 & 0.0867 & 0.0988 & 0.1385 \\
\hline ROW 3 & 0.298 & 0.1165 & 1.000 & 0.1334 & 0.0457 & 0.1838 \\
\hline ROW 4 & 0.071 & 0.0867 & 0.133 & 1.000 & $\mathbf{0 . 6 2 2 1}$ & -0.1165 \\
\hline ROW 5 & 0.055 & 0.0988 & 0.046 & 0.6221 & 1.000 & $\mathbf{0 . 4 9 8 0}$ \\
\hline ROW 6 & 0.161 & 0.1385 & 0.184 & -0.117 & 0.498 & 1.0000 \\
\hline
\end{tabular}

При формулировке Критерия 1 будем опираться на известные применяемые факты. По определению формулы коэффициентов парной корреляции $\quad \mathrm{r}_{\mathrm{ij}}=\operatorname{corr}\left(\mathrm{z}_{\mathrm{i}}, \mathrm{z}_{\mathrm{j}}\right), \quad \mathrm{c}_{\mathrm{ij}}=\operatorname{corr}\left(\mathrm{z}_{\mathrm{i}}, \mathrm{y}_{\mathrm{j}}\right)$ представляют собой косинусы углов между 131мерными векторами $\mathrm{z}_{\mathrm{i}}=\left(\mathrm{z}_{1, \mathrm{i}}, \ldots, \mathrm{z}_{131, \mathrm{i}}\right)^{\mathrm{T}}$, $\mathrm{z}_{\mathrm{j}}=\left(\mathrm{z}_{1 \mathrm{j}}, \ldots, \mathrm{z}_{131, \mathrm{j}}\right)^{\mathrm{T}}, \quad \mathrm{y}_{\mathrm{j}}=\left(\mathrm{y}_{1 \mathrm{j}}, \ldots, \quad \mathrm{y}_{131, \mathrm{j}}\right)^{\mathrm{T}}, \quad \mathrm{i}, \mathrm{j}=1, \ldots, 6$. Равенство $\quad \mathrm{r}_{4,5}=0.6221$ выражает заметную степень выраженности линейной связи, но она не выявляет причину связи. Из-за воздействия макроэкономических факторов оба показателя (№1 и №4) возрастают или уменьшаются на протяжении коротких периодов времени, но рост одного из них не является причиной роста другого. Всюду ниже при формулировке фраз относительно ФИ (НФИ) для упрощения текста вместо фразы «риск изменения процентной ставки типа «доходность к дате погашения» ФИ №j» будем писать «риск ФИ №j».

Для выявления номеров интервалов, содержащих значения доходностей ГЦБ с высокими рисками мы используем вывод, приведенный выше: наибольшее собственное число $\lambda_{1}=1+\mathrm{r}_{12}$ тем больше, чем больше коэффициент корреляции $\mathrm{r}_{12}$. Содержательно это соответствует утверждению «большое значение риска НФИ обеспечивается большими значениями коэффициентов корреляции между доходностями ФИ». По их оценке силы связи коэффициентов корреляции обычно используется шкала Чеддока (Chad-dock scale). По этой шкале количественная мера тесноты связи: абсолютное значение коэффициента, принадлежащее интервалу от 0 до 0.3 - качественно интерпретируется как «очень слабая», интервалу от 0.3 до 0.5-«слабая, умеренная», интервалу от 0.5 до 0.7 - «заметная» (mode-rate positive), интервалу от 0.7 до 0.9 - «высокая», интервалу от 0.9 до 1- «очень высокая».

\section{Критерии выделения коррелированных высокорисковых финансовых инструментов.

Выделим «заметные» значения
коэффициентов парной корреляции из элементов, расположенных выше диагонали симметрической кореляционной матрицы $\mathrm{R}_{66}$. Большинство ее элементов по качественной интерпретации меры тесноты связи интерпретируются как «очень слабая», «слабая». «Заметная» теснота связи выражена только между 1-ым и 2-ым ФИ $\mathrm{r}_{1,2}=0.6654$. только между 4-ым и 5-ым ФИ $\mathrm{r}_{4,5}=0.6221$ и только между 5-ым и 6-ым ФИ $\mathrm{r}_{5,6}=0.4987 \approx 0.5$. Остальные коэффициенты кореляции не отражают (по шкале Чеддока) степень заметной силы связи. Пары индексов $(1,2), \quad(4,5), \quad(5,6)$ высоких коэффициентов корреляции $\mathrm{r}_{12}=0.6654, \mathrm{r}_{45}=0.6221, \quad \mathrm{r}_{56}=0.4980$ указывают на наличие высокорисковых ГЦБ, у которых «доходности к дате погашения» принадлежат интервалам №1, №2, №4, №5, №6 временной структуры. Заметим, что интервал №3 отсутствует в этом списке выделенных по Критерию 1 пары индексов $(\mathrm{k}, \mathrm{j})$ доминирующих по абсолютной велмчине коэффициентов корреляции. Это отсутствие означает незаметное значение рисковости у ФИ №3, что противоречит банковской практике. Ниже ФИ №3, характеризуемый доходностями из интервала №3, будет включен по Критерию 2 в один из локальных высокорисковых портфелей.

Рассматриваемые ФИ достаточно «заметно» связаны внутри своего кластера: ФИ №1 коррелирует с ФИ №2, ФИ №4 достаточно высоко коррелирует с ФИ №5, а ФИ №5 заметно коррелирует с ФИ №6. Этим мы выявили по матрице выборочных коэффициентов корреляции неполный состав высокорисковых ФИ, которые, как показано ниже, делятся в зависимости от 
номера интервала временной структуры на 3 кластера новых финансовых инструментов: НФИ №1 ГЦБ, НФИ №2 ГЦБ, НФИ №3 ГЦБ. Это разбиение корректно проведено с применением модели анализа главных компонент. Численно решена прямая задача АГК (ПЗ АГК [1,3]), входным объектом является стандартизованная выборка $Z_{131,6}$, элементы которой вычислены из элементов матрицы исходных данных $\mathrm{X}_{131,6}^{\circ}$ Стандартизация матрица $\mathrm{Z}_{131,6}$ позволяет сопоставлять друг с другом степени рисковости изменения доходности указанного нами типа у коротких, краткосрочных, среднесрочных, долгосрочных ГЦБ. Выборка $Z_{131,6}$ является одним из ассоциированных решений (O3 АГК)[2,3]: $\quad \Lambda_{66}=>\left(\mathrm{C}^{(\ell)}{ }_{66}, \mathrm{R}_{66}{ }^{(\ell)}, \quad \mathrm{Y}_{131,6}, \quad \mathrm{Z}^{(\ell)}{ }_{131,6}\right)$, $\ell=1, \ldots, \infty$.

Сформулируем критерий вычисления пар $(\mathrm{i}, \mathrm{j})$ номеров интервалов временной структуры, для которых i-ые и j-ые временные ряды $\mathbf{Z}_{\mathrm{i}}=\left(\mathrm{z}_{1 \mathrm{i}}, \ldots, \mathrm{z}_{131, \mathrm{i}}\right)^{\mathrm{T}}, \mathbf{Z}_{\mathbf{j}}=\left(\mathrm{z}_{1 \mathrm{j}}, \ldots, \mathrm{z}_{131, \mathrm{j}}\right)^{\mathrm{T}} \quad$ определяют значения коэффициентов корреляций $\mathrm{r}_{\mathrm{ij}}=\operatorname{corr}\left(\mathrm{z}_{\mathrm{i}}, \mathrm{z}_{\mathrm{j}}\right), \quad$ удовлетворяют критерию «заметной» связи по шкале Чаддока, т.е. соответствуют доходностям ГЦБ с высокими рисками.

Критерий 1. Пары индексов $(\mathrm{k}, \mathrm{j}), \mathrm{k}<\mathrm{j}$, доминирующих по абсолютной величине коэффициентов корреляции удовлетворяют условию $\quad \ell=\max \left\{\mathrm{j}: \operatorname{abs}\left(\mathrm{r}_{\mathrm{kj}}\right)=\operatorname{abs}\left(\operatorname{corr}\left(\mathrm{z}_{\mathrm{K}}, \mathrm{z}_{\mathrm{j}}\right)\right)>\mathrm{r}_{0}\right.$, $\left.\mathrm{kC}\{1, \ldots, 6\}, \mathrm{R}_{66}=\left(\mathrm{r}_{\mathrm{ij}}\right)\right\}$.

Фиксируем пороговое значение: $\mathrm{r}_{0}=0.5000$. Оно соответствует выбору силы связи коэффициентов корреляции по шкале Чеддока: от 0.5 до 0.7 (заметная, средняя). Это - результат анализа домирирующих значений парных коэффициентов корреляций $\mathrm{r}_{\mathrm{ij}}=\operatorname{corr}\left(\mathrm{z}_{\mathrm{i}}, \mathrm{z}_{\mathrm{j}}\right) \geq \mathrm{r}_{0}$. При $\mathrm{r}_{0}=0.5000$ число доминирующих по абсолютной величине коэффициентов корреляции, расположенных выше диагонали матрицы $\mathrm{R}_{66}$ равно 3.

Финансовая интерпретация собственных векторов. Далее мы проведем анализ элементов матрицы собственных векторов $\mathrm{C}_{66}$, вычисленных для проанализированной нами матрицы коэффициентов корреляций $\mathrm{R}_{66}$. для этого имеются следующие существенные основания. Для портфеля независимых ФИ риск изменения рассматриваемого нами типа процентной ставки измеряется суммой дисперсий соответствующих некоррелированных нормированных значений процентных ставок временных рядов. Но наши временные $\quad$ яяды $\quad\left\{\mathrm{z}_{1, \mathrm{j}}, \mathrm{z}_{2, \mathrm{j}}, \ldots, \mathrm{z}_{131, \mathrm{j}}\right\}, \mathrm{j}=1, \ldots, 6$, нормированных значений процентных ставок заметно коррелированны между собой по Критерию 1.

Преобразуем, как рекомендовано в работах [5-9] 6 z-переменных в 6 у-переменные, но так, чтобы сумма дисперсий не изменилась. Для доходностей 6 ФИ их сумма дисперсий равна 6 . при этом все $\mathrm{z}$-переменные имеют одинаковые дисперсии, равные 1 . Такие $\mathrm{z}$-переменные нас не устраивают, ибо мы знаем, что существуют какие-то подмножества ФИ-ов, у которых риски будут большими, средними, малыми. Мы выбрали в качестве измерителя степени риска дисперсию временного ряда, состоящего из значений избыточных доходностей ГЦБ к дате погашения. Применим ортогональное преобразование - матрицу $\mathrm{C}_{66}$, такую, что $\mathrm{C}_{66}^{\mathrm{T}} \mathrm{C}_{66}=\mathrm{I}_{66}, \mathrm{C}_{66} \mathrm{C}_{66}^{\mathrm{T}}{ }_{66}=\mathrm{I}_{66}$, к матрице $\mathrm{z}$-переменных $\mathrm{Z}_{131,6}$ : $\quad \mathrm{Y}_{131,6}=\mathrm{Z}_{131,6} \mathrm{C}_{66}$. Ортогональное преобразование $\mathrm{C}_{66}$ не изменяет сумму дисперсий z-переменных:

$\mathrm{R}_{66}=(1 / 131) \mathrm{Z}_{131,6}^{\mathrm{T}} \mathrm{Z}_{16,4}: \mathrm{Z}_{131,6}=>\left(\mathrm{R}_{66}, \mathrm{C}_{66}, \Lambda_{66}, \mathrm{Y}_{131,6}\right)$.

Как найти матрицу $\mathrm{C}_{66}$ ? Одним из типов матриц $\mathrm{C}_{66}$, обладающих нужными нам свойствами, является матрица собственных векторов $\quad \mathbf{c}_{\mathbf{j}}=\left(\mathrm{c}_{1 \mathrm{j}}, \mathrm{c}_{2 \mathrm{j}} \ldots \mathrm{c}_{\mathrm{nj}}\right)^{\mathrm{T}}, \quad$ образующих ортогональную матрицу $\mathrm{C}_{66}=\left[\mathbf{c}_{1}\left|\mathbf{c}_{2}\right| \quad \ldots \mid \mathbf{c}_{6}\right]$, согласованную с матрицей собственных чисел $\Lambda_{66}=\operatorname{diag}\left(\lambda_{1}, \lambda_{2}, \ldots, \lambda_{6}\right), \lambda_{1}>\ldots>\lambda_{6}>0$, таким образом, что $\quad \mathrm{R}_{66} \mathrm{C}_{66}=\mathrm{C}_{66} \Lambda_{66}, \quad \mathrm{C}_{66}^{\mathrm{T}} \mathrm{C}_{66}=\mathrm{C}_{66} \mathrm{C}_{66}^{\mathrm{T}}=\mathrm{I}_{66}, \quad$ где $\operatorname{diag}\left(\mathrm{R}_{66}\right)=(1, \ldots, 1), \operatorname{tr}\left(\mathrm{R}_{66}\right)=1+1+1+1+1+1=\operatorname{tr}\left(\Lambda_{66}\right)=$ $=\lambda_{1}+\ldots+\lambda_{6}=6$. Матрицы $\mathrm{C}_{66}$ и $\Lambda_{66}$ вычисляются одновременно по известной корреляционной матрице $\mathrm{R}_{66}$. Матрица $\mathrm{R}_{66}$ вычисляется по стандартизованной выборке

$$
\mathrm{Z}_{131,6}: \mathrm{R}_{66}=(1 / 131) \mathrm{Z}^{\mathrm{T}}{ }_{131,6} \mathrm{Z}_{131,6} \text {. }
$$

Докажем, что матрица $\mathrm{C}_{66}$ является корреляционной матрицей и, аналогично матрице $\mathrm{R}_{66}=(1 / 131) \mathrm{Z}^{\mathrm{T}}{ }_{131,6} \mathrm{Z}_{131,6}$, вычисляется в виде матрицы, равной произведению транспонированной матрицы $\mathrm{Z}_{131,6}$ стандартизованных Z-переменных на матрицу стандартизованных у-переменных $\mathrm{Y}_{131,6} \Lambda^{-1}$. По этому определению

$$
\begin{aligned}
& (1 / \mathrm{m}) \mathrm{Z}^{\mathrm{T}}{ }_{131,6}\left(\mathrm{Y}_{131,6} \Lambda^{(-1)}{ }_{66}\right)=(1 / \mathrm{m}) \mathrm{Z}^{\mathrm{T}}{ }_{131,6} \mathrm{Z}_{131,6} \mathrm{C}_{66} \\
& \left.\Lambda^{(-1)}{ }_{66}\right)=\mathrm{R}_{66} \mathrm{C} \Lambda^{(-1)}{ }_{66} \cdot \\
& \text { Так как матрица } \mathrm{C}_{66} \text { является матрицей }
\end{aligned}
$$
собственных векторов, то верно равенство $\mathrm{R}_{66} \mathrm{C}_{66}=\mathrm{C}_{66} \Lambda_{66}$ Подставляя это равенство в нашу формулу имеем:

$$
\mathrm{R}_{66} \mathrm{C}_{66} \Lambda^{(-1)}{ }_{66}=\mathrm{C}_{66} \Lambda_{66} \Lambda^{(-1)}{ }_{66}=\mathrm{C}_{66} \mathrm{I}_{66}=\mathrm{C}_{66} \text {. }
$$

Мы доказали равенство

$$
(1 / \mathrm{m}) \mathrm{Z}^{\mathrm{T}}{ }_{131,6}\left(\mathrm{Y}_{131,6} \Lambda^{(-1)}{ }_{66}\right)=\mathrm{C}_{66} \text {. }
$$

Аналогично вычисляем матрицу $\mathrm{C}^{\mathrm{T}} 66$ корреляций между 2-мя множествами переменных, умножив число $(1 / \mathrm{m})$ на произведение одной матрицы $\mathrm{Y} \Lambda^{(-1)}$ стандартизованных у-переменных на матрицу $\mathrm{Z}_{\mathrm{mn}}$ стандартизованных Z-переменных:

$$
\begin{gathered}
(1 / \mathrm{m})\left(\mathrm{Y} \Lambda^{(-1)}{ }_{66}\right)^{\mathrm{T}} \mathrm{Z}=\left(\Lambda^{(-1)}{ }_{66}\right)(1 / \mathrm{m})\left(\mathrm{Y}^{\mathrm{T}}\right) \mathrm{Z}= \\
=\left(\Lambda^{(-1)}{ }_{66}\right)(1 / \mathrm{m})\left(\mathrm{ZC} \mathrm{C}_{66}\right)^{\mathrm{T}} \mathrm{Z}=\left(\Lambda^{(-1)}{ }_{66}\right) \mathrm{C}^{\mathrm{T}}{ }_{66}(1 / \mathrm{m}) \mathrm{Z}^{\mathrm{T}} \mathrm{Z}= \\
=\left(\Lambda^{(-1)}{ }_{66}\right) \mathrm{C}^{\mathrm{T}}{ }_{66} \mathrm{R}_{66}=\left(\Lambda^{(-1)}{ }_{66}\right) \Lambda{ }_{66} \mathrm{C}^{\mathrm{T}}{ }_{66}=\mathrm{C}^{\mathrm{T}}{ }_{66} .
\end{gathered}
$$

Докажем обратное утверждение: если ортогональная матрица $\mathrm{C}_{66} \quad$ является корреляционной матрицей пар (z,y)-переменных: $\mathrm{C}_{66}=(1 / 131) \mathrm{Z}^{\mathrm{T}}{ }_{131,6} \mathrm{Y}_{131,6} \Lambda^{-1}{ }_{66}, \quad$ то она-матрица 
собственных векторов для корреляционной матрицы $\mathrm{R}_{66}$. Пусть верно равенство $\mathrm{C}_{66}=(1 / 131) \mathrm{Z}_{131,6}^{\mathrm{T}} \quad \mathrm{Y}_{131,6} \Lambda^{-1}$, где $\mathrm{y}$-переменные стандартизируются для корректного вычисления коэффициентов коррляции Пирсона. Докажем, что так определенная матрица $\mathrm{C}_{66}$ являтся матрицей собственных векторов для корреляционной матрицы $\mathrm{R}_{66}$ и сохраняет свойство ортогонормированности: $\mathrm{C}^{\mathrm{T}}{ }_{66} \mathrm{C}_{66}=\mathrm{I}_{66}$, $\mathrm{C}_{66} \mathrm{C}_{66}^{\mathrm{T}}=\mathrm{I}_{66}$. Подставляя в эти формулы вышеприведенное значение $\mathrm{C}_{66}$ имем: $\mathrm{C}^{\mathrm{T}}{ }_{66} \mathrm{C}_{66}=(1 / 131)\left(\mathrm{Z}^{\mathrm{T}} \mathrm{Y} \Lambda^{-1}\right)^{\mathrm{T}}(1 / 131) \mathrm{Z}^{\mathrm{T}} \mathrm{Y} \Lambda^{-1}=$ $=\left(\Lambda^{-1} \mathrm{Y}^{\mathrm{T}} \mathrm{Z}\right)(1 / 131)(1 / 131) \mathrm{Z}^{\mathrm{T}} \mathrm{Y} \Lambda^{-1}=$ $=\left(\Lambda^{-1}(1 / 131) \mathrm{C}^{\mathrm{T}} \mathrm{Z}^{\mathrm{T}} \mathrm{Z}\right)(1 / 131) \mathrm{Z}^{\mathrm{T}} \mathrm{ZC} \Lambda^{-1}=$ $=\Lambda^{-1} \mathrm{C}^{\mathrm{T}} \mathrm{RRC}_{66} \Lambda^{-1}=\Lambda^{-1} \Lambda \mathrm{C}^{\mathrm{T}} \mathrm{C} \Lambda \Lambda^{-1}=$ $=\Lambda^{-1} \Lambda \mathrm{I} \Lambda \Lambda^{-1}=\mathrm{I}$. Аналогично доказывается равенство $\mathrm{C}_{66} \mathrm{C}_{66}^{\mathrm{T}}=\mathrm{I}_{66}$. Формула $\mathrm{C}_{66}=(1 / 131)$ $\mathrm{Z}^{\mathrm{T}}{ }_{131,6} \mathrm{Y}_{131,6} \Lambda^{-1}$ не нарушает свойство согласованности собственных векторов (из столбцов матрицы $\left.\mathrm{C}_{66}=(1 / 131) \mathrm{Z}^{\mathrm{T}}{ }_{131,6} \mathrm{Y}_{131,6} \Lambda^{-1}\right)$ и собственных чисел $\quad \Lambda_{66}=\operatorname{diag}\left(\lambda_{1}, \ldots, \lambda_{6}\right)$ корреляционной матрицы $\mathrm{R}_{66}: \quad \mathrm{R}_{66} \mathrm{C}_{66}=\mathrm{C}_{66} \Lambda_{66}$. Соблюдение этого свойства видно из соотношений: $\quad \mathrm{R}_{66} \mathrm{C}_{66}=\mathrm{R}_{66}(1 / 131) \quad \mathrm{Z}^{\mathrm{T}}{ }_{131,6} \mathrm{Y}_{131,6}$ $\Lambda^{-1}=\mathrm{R}_{66} \mathrm{C}_{66}(1 / 131) \mathrm{Y}^{\mathrm{T}}{ }_{131,6} \mathrm{Y}_{131,6} \Lambda^{-1}=\mathrm{R}_{66} \mathrm{C}_{66}$.

Аналогично, подставляя значение $\mathrm{C}_{66}=(1 / 131)$ $\mathrm{Z}^{\mathrm{T}}{ }_{131,6} \mathrm{Y}_{131,6} \Lambda^{-1}$ в правую часть равенства $\mathrm{R}_{66} \mathrm{C}_{66}=$ $\mathrm{C}_{66} \Lambda_{66}$ имеем: $\mathrm{C}_{66} \Lambda_{66}=(1 / 131) \mathrm{Z}^{\mathrm{T}}{ }_{131,6} \mathrm{Y}_{131,6} \Lambda^{-1} \Lambda_{66}=$ $\mathrm{C}_{66}(1 / 131) \mathrm{Y}_{131,6}^{\mathrm{T}} \mathrm{Y}_{131,6} \Lambda^{-1} \Lambda_{66}=\mathrm{C}_{66} \Lambda_{66}$.

Новые у-переменные равны линейным комбинациям 6 z-переменных и не коррелируют друг с другом. При этом сумма дисперсий всех у-переменных равна 6 , т.е. след матрицы $\mathrm{R}_{66}$ равен 6: $\operatorname{trace}\left(\mathrm{R}_{66}\right)=\operatorname{trace}\left(\Lambda_{66}\right)=(1,8076+1,7936+$ $1.1509+0.8555+0.2887+0.1037)=6$. Но дисперсии новых у-переменных упорядочены в порядке убывания: $\lambda_{1}>\ldots>\lambda_{6}>0$, и наибольшие по значению дисперсии $\lambda_{1}>\ldots>\lambda_{\ell}$ равны значениям рисков изменения $\ell$ линейных комбинаций. Каждую линейную комбинацию из 6 zпеременных интерпретируем как независимый новый финансовый инструмент (НФИ), составленный из комбинации только высокорисковых зависимых ФИ.

\section{Критерий выделения числа} некоррелированных высокорисковых НФИ. Аналогично ФИ №3, который по Критерию 1 не был включен в число высокорисковых ФИ, среди 6 НФИ могут встретиться низкорисковые НФИ. Измерителем степени рисковости НФИ является его дисперсия $\lambda_{\mathrm{j}, \mathrm{j}}=1, \ldots, 6$. Так как собственные числа корреляционной матрицы $\mathrm{R}_{66}$ упорядочены в порядке убывания их величин: $\lambda_{1}=1.8076$, $\lambda_{2}=1.7936, \quad \lambda_{3}=1.1509, \quad \lambda_{4}=0.8555, \quad \lambda_{5}=0.2887$, $\lambda_{6}=0,1037$, в сумме равных 6 , то выберем доминирующее количество $\mathrm{L} \geq 1$ собственных чисел, соответствующих L у-переменным (мы поставили им в соответствие НФИ). В других, отличных от нашей, задачах для определения числа L обоснованно применяются критерии Кайзера-Дикмана [25], Тэрстоуна [25], Кателла [25], Хорна [26], Джоллиффа [27]. Эти критерии используются для определения числа $\mathrm{L}$ существенных в том или ином смысле упеременных, доля которых превышает некоторое фиксированное пороговое значение. По критерию высокорисковости значение дисперсии $\mathrm{y}$ переменных $\lambda_{\mathrm{L}}$ должно быть как можно большим, а меньшее из доминирующих значений $\lambda_{1}, \ldots, \lambda_{\mathrm{L}}$ должно быть таковым, что в соответствующем ему собственном векторе $\mathbf{c}_{. L}=\left(\mathrm{c}_{1 \mathrm{~L}}, \mathrm{c}_{2 \mathrm{~L}}, \mathrm{c}_{3 \mathrm{~L}}, \quad \ldots, \mathrm{c}_{6 \mathrm{~L}}\right)^{\mathrm{T}} \quad$ присутствовал бы коэффициент корреляции $\mathrm{c}_{3 \mathrm{~L}}=\operatorname{corr}\left(\mathrm{z}_{3}, \mathrm{y}_{\mathrm{L}}\right) \geq \mathrm{c}_{0}=0.4631 \approx 0.5, \quad$ с $\quad$ высоким значением коэффициента корреляции при $\mathrm{L}=3$ $\mathrm{c}_{33}=\operatorname{corr}\left(\mathrm{z}_{3}, \mathrm{y}_{3}\right)=0.4631 \approx 0.5 \geq 0.5$, показывающим на наличие высокорисковых ГЦБ со значениями процентных ставок типа «доходность к дате погашения», принадлежащих интервалу №3 временной структуры.

Значение $\mathrm{c}_{33}=\operatorname{corr}\left(\mathrm{z}_{3}, \mathrm{y}_{3}\right)=0.4631 \approx 0.5$ при $\mathrm{L}=3$ оказалось редким в множестве значений элементов $\mathrm{c}^{(\ell)}{ }_{33}$ сгенерированных выше матриц $\mathrm{C}^{(\ell)}{ }_{66 . \ell} \ell=1, \ldots, 1000$. Эти матрицы $\mathrm{C}^{(\ell)}{ }_{66}$ выше моделировались как решения ОС3 $\Lambda_{66}=>\left(\mathrm{C}^{(\ell)}{ }_{66}, \mathrm{R}^{(\ell)}{ }_{66}\right), \ell=1, \ldots, 1000 . \quad$ Программа CORMAP [22] реализует алгоритм Чалмерса [23] и применяется выше при вычислении матриц $\mathrm{R}^{(\ell)}{ }_{66}=\mathrm{C}^{(\ell)}{ }_{66} \Lambda_{66} \mathrm{C}^{(\ell) \mathrm{T}}{ }_{66}, \ell=1, \ldots, 1000, \quad$ причем неравенство вида $\mathrm{c}^{(\ell)}{ }_{33} \geq 0.5$ выполнялось много раз, что подтверждает статистическую достоверность неравенства $\mathrm{c}_{33} \geq 0.5$.

Критерий 2. Число собственных векторов $\ell_{\mathrm{ev}}$ равно числу пар индексов $\left(\mathrm{k}, \ell_{\mathrm{ev}}\right)$ доминирующих по абсолютной величине компонентов $c_{\mathrm{k} \ell}=\operatorname{corr}\left(\mathrm{z}_{\mathrm{k}}, \mathrm{y} \ell\right), \quad \ell=\ell_{\mathrm{ev}}$, собственных векторов(eigen- vectors), расположенных в столбцах матрицы $\mathrm{C}_{66}$, и соответствующих

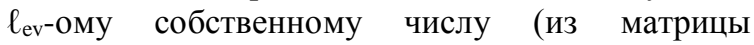
собственных чисел $\left.\Lambda_{66}=\operatorname{diag}\left(\lambda_{1}, \ldots \lambda_{6}\right)\right)$, у которых хотя бы одна $\left(\mathrm{k}, \ell_{\mathrm{ev}}\right)$-компонента удовлетворяет условию

$\ell_{\mathrm{ev}}=\max \left\{\mathrm{j}: \operatorname{abs}\left(\mathrm{c}_{\mathrm{kj}}\right)>\mathrm{c}_{0}, \mathrm{k} \in\{1, \ldots, 6\}, \mathrm{C}_{66}=\left[\mathbf{c}_{1}\left|\mathbf{c}_{2}\right| \ldots \mid \mathbf{c}_{6}\right]\right.$, $\left.\mathbf{c}_{\mathrm{j}}=\left(\mathrm{c}_{1 \mathrm{j}}, \ldots, \mathrm{c}_{\mathrm{kj}}, \ldots, \mathrm{c}_{6 \mathrm{j}}\right)^{\mathrm{T}}, \lambda_{1}, \geq \ldots \geq \lambda_{\mathrm{j}} \geq \ldots \geq \lambda_{6}, \lambda_{1}+\ldots+\lambda_{6}=6\right\}$.

Здесь пороговое значение $\mathrm{r}_{0}$ из Критерия 1 мы полагаем равным пороговому значению $\mathrm{c}_{0}$ из Критерия 2: $\mathrm{r}_{0}=\mathrm{c}_{0}=0.500$, что предполагает «равносторонний треугольник» степеней связей между парами из 3 переменных. Возможно, что имеются другие соотношения между значениями $\mathrm{r}_{0}$ и $\mathrm{c}_{0}$, но это - другая задача.

Таким образом, коэффициент корреляции $\mathrm{c}_{3 \ell}=\operatorname{corr}\left(\mathrm{z}_{3}, \mathrm{y}_{3}\right)$, удовлетворящий Критерию 2 (значения $\mathrm{r}_{34}=0.1334, \mathrm{r}_{35}=0.0457, \mathrm{r}_{36}=0.1838$ не удовлетворяют Критерию 1), ибо выделена его пара индексов (3,3). Доминирующий по абсолютной величине квадрат коэффициента 
корреляции $\mathrm{c}^{2}{ }_{33}=\operatorname{corr}\left(\mathrm{z}_{3}, \mathrm{y}_{3}\right)$ отражает «заметную» степень рисковости ФИ №3, характеризуемого доходностями из интервала №3, и включен по Критерию 2 в НФИ №3. Таким правым индексом $\mathrm{L}=3$ компоненты $\mathrm{c}_{3 \mathrm{~L}}$ собственного вектора номер $\mathrm{L}, \mathbf{c}_{\mathbf{L}}=\left(\mathrm{c}_{1 \mathrm{~L}}, \mathrm{c}_{2 \mathrm{~L}}, \mathrm{c}_{3 \mathrm{~L}}, \ldots, \mathrm{c}_{6 \mathrm{~L}}\right)^{\mathrm{T}}$ в нашем случае является собственный

вектор

№3:

$\mathbf{c}_{.3}=\left(\mathrm{c}_{13}, \mathrm{c}_{23}, \mathrm{c}_{33}, \ldots, \mathrm{c}_{63}\right)^{\mathrm{T}}=(0.1233,-0.0859,0.4631$,

$-0.5614, \quad-0.0852,0.6637)^{\mathrm{T}}$. При наличии нескольких интервалов, номера которых обладают таким же свойством, что и приведенный выше индекс 3 компоненты вектора №3 находим их аналогичным образом.

Матрица собственных векторов $\mathbf{C}_{66}$

Таблица 2

\begin{tabular}{|l|l|l|l|l|l|l|}
\hline COLUMN & \multicolumn{1}{|c|}{$\mathbf{1}$} & \multicolumn{1}{|c|}{$\mathbf{2}$} & \multicolumn{1}{c|}{$\mathbf{3}$} & \multicolumn{1}{c|}{4} & \multicolumn{1}{c|}{5} & \multicolumn{1}{c|}{6} \\
\hline ROW 1 & $\mathbf{0 . 6 5 2 9}$ & -0.1771 & 0.1233 & 0.1204 & -0.6983 & 0.1585 \\
\hline ROW 2 & $\mathbf{0 . 6 3 7 9}$ & 0.0606 & -0.0859 & 0.3787 & 0.6622 & 0.0014 \\
\hline ROW 3 & 0.3378 & 0.1535 & $\mathbf{0 . 4 6 3 1}$ & -0.7574 & 0.1822 & -0.2023 \\
\hline ROW 4 & 0.1643 & $\mathbf{0 . 5 1 4 2}$ & $\mathbf{- 0 . 5 6 1 4}$ & -0.3126 & -0.0064 & 0.5438 \\
\hline ROW 5 & 0.1037 & $\mathbf{0 . 6 9 4 0}$ & -0.0852 & 0.2567 & -0.1930 & -0.6302 \\
\hline ROW 6 & -0.1222 & 0.4420 & $\mathbf{0 . 6 6 3 7}$ & 0.3237 & 0.0581 & 0.4910 \\
\hline
\end{tabular}

Выделим только «заметные» значения рисков ФИ из левой части и «заменяющих» их в будущем «заметные» значения рисков ФИ из правой части интервалов временной структуры (таблица 4). Анализ таблицы 4 показывает настоящую и перспективную привлекательности для инвесторов не только «заметных» в данный момент ФИ, но всех остальных ФИ. Остающая часть риска НФИ №2, а именно

$100 \%-74,6038 \%=25,39 \%$, состоит из рисков ГЦБ (например, $\mathrm{c}^{2}{ }_{25}=0.6622^{2}=0.4385$ для ФИ №2), которые возрастут потом при изменении (из 5-го к 1-ому) интервала временной структуры в последующие моменты времени, при уменьшении их сроков до даты погашения. Для НФИ №1 доля рисков таких «заметных» ГЦБ равна $100 \%-83,32 \%=16,68 \%$, для НФИ №3-100\%$65.496 \% \%=34.504 \%$. Доли «заметных» рисков 83.32\%.74.6038\%.65.496\% трех НФИ объяснены

\begin{tabular}{|c|c|c|c|c|c|c|}
\hline & $\begin{array}{c}\mathrm{c}^{2}{ }_{\mathrm{i} 1}= \\
\operatorname{corr}\left(\mathrm{z}_{\mathrm{i}}, \mathrm{y}_{1}\right)\end{array}$ & $\begin{array}{c}\mathrm{c}^{2}{ }_{\mathrm{i} 2}= \\
\operatorname{corr}\left(\mathrm{z}_{\mathrm{i}}, \mathrm{y}_{2}\right)\end{array}$ & $\begin{array}{c}\mathrm{c}^{2}{ }_{\mathrm{i} 3}= \\
\operatorname{corr}\left(\mathrm{z}_{\mathrm{i}}, \mathrm{y}_{3}\right) \\
\end{array}$ & $\begin{array}{c}\mathrm{c}^{2}{ }_{\mathrm{i} 4}= \\
\operatorname{corr}\left(\mathrm{z}_{\mathrm{i}}, \mathrm{y}_{4}\right)\end{array}$ & $\begin{array}{c}\mathrm{c}^{2}{ }_{\mathrm{i} 5}= \\
\operatorname{corr}\left(\mathrm{z}_{\mathrm{i}}, \mathrm{y}_{5}\right) \\
\end{array}$ & $\begin{array}{c}\mathrm{c}^{2}{ }_{\mathrm{i} 6}= \\
\operatorname{corr}\left(\mathrm{z}_{\mathrm{i}}, \mathrm{y}_{6}\right)\end{array}$ \\
\hline ФИ №1 & 0.4263 & 0.0314 & 0.0152 & 0.0145 & 0.4876 & 0.0251 \\
\hline ФИ №2 & 0.4069 & 0.0037 & 0.0074 & 0.1434 & 0.4385 & 0.0000 \\
\hline ФИ №3 & 0.1141 & 0.0236 & 0.2145 & 0.5737 & 0.0332 & 0.0409 \\
\hline ФИ №4 & 0.0270 & 0.2644 & 0.3152 & 0.0977 & 0.0000 & 0.2957 \\
\hline ФИ №5 & 0.0108 & 0.4816 & 0.0073 & 0.0659 & 0.0372 & 0.3972 \\
\hline ФИ №6 & 0.0149 & 0.1954 & 0.4405 & 0.1048 & 0.0034 & 0.2411 \\
\hline \multicolumn{7}{|c|}{ Распределение приемлемых для дилеров и игнорируемых значений долей рисков ФИ в долях НФИ } \\
\hline$\sum$ & $83.32 \%$ & $74.60 \%$ & $97.01 \%$ & $71.71 \%$ & $92.61 \%$ & $93.40 \%$ \\
\hline $100-\sum$ & $16.68 \%$ & $25.40 \%$ & $2.99 \%$ & $28.29 \%$ & $7.39 \%$ & $6.60 \%$ \\
\hline & $100.00 \%$ & $100.00 \%$ & $100.00 \%$ & $100.00 \%$ & $100.00 \%$ & $100.00 \%$ \\
\hline
\end{tabular}

и пригодны для практического применения дилерами банка, а риски: $16.68 \%, 25.39 \%$, $34.504 \%$, - относятся к ГЦБ с низкими доходностями, в данный момент времени $\mathrm{t}$ не привлекательных для инвесторов. Но как показывают строки 1 и 2 Таблицы 4 ФИ №1, ФИ №2 с значениями рисков $0.4876,0.4385$ (в них направлены концы стрелок) входят в НФИ №5, а значения рисков ФИ №1, ФИ №2 (0.4263 и 0,4069 - из них исходят стрелки) в НФИ №1 заменятся на новые, ибо их вновь вычисленные в будущем по отношению к текущему моменту времени $\mathrm{t}$ изотермные ряды будут находиться в столбцах №1, №2 будущей матрицы $\mathrm{X}^{0}{ }_{131,6}$. Аналогично 


\begin{tabular}{|c|c|c|c|c|c|c|}
\hline Impact Factor: & $\begin{array}{l}\text { ISRA (India) } \\
\text { ISI (Dubai, UAE } \\
\text { GIF (Australia) } \\
\text { JIF }\end{array}$ & $\begin{array}{l}=1.344 \\
=0.829 \\
=0.564 \\
=1.500\end{array}$ & $\begin{array}{l}\text { SIS (USA) } \\
\text { PИНЦ (Russia) } \\
\text { ESJI (KZ) } \\
\text { SJIF (Morocco) }\end{array}$ & $\begin{array}{l}=0.912 \\
=\mathbf{0 . 2 0 7} \\
=\mathbf{3 . 8 6 0} \\
=\mathbf{2 . 0 3 1}\end{array}$ & $\begin{array}{l}\text { ICV (Poland) } \\
\text { PIF (India) } \\
\text { IBI (India) }\end{array}$ & $\begin{array}{l}=6.630 \\
=1.940 \\
=4.260\end{array}$ \\
\hline
\end{tabular}

интерпретируются стрелки в других строках Таблицы 4. Это - наглядная иллюстрация повышенной привлекательности реального портфеля ГЦБ, наблюдаемой на практике.

Приведенные демонстрируют практике.

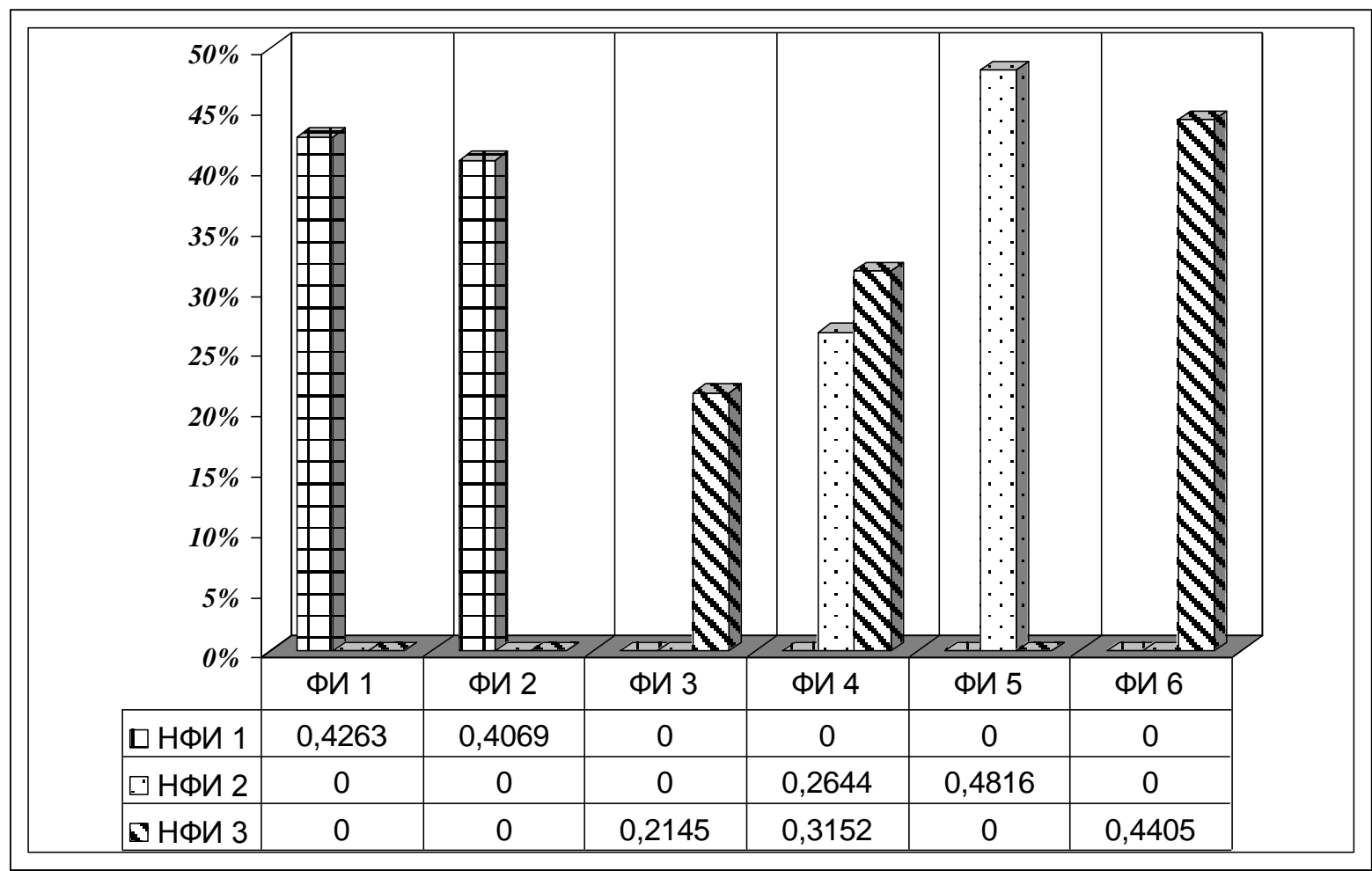

Рисунок 1 - Содержание в 3 НФИ вычисленных значений (долей) рисков изменения доходностей типа «доходность к дате погашения» «заметных» ФИ.

Заметим: матрица собственных векторов $\mathrm{C}_{66}$ и диагональная матрица $\Lambda_{66}=\operatorname{diag}\left(\lambda_{1, \ldots,} \lambda_{6}\right)$ собственных чисел (пара матриц) являются решением однородной спектральной задачи $\mathrm{RC}=\mathrm{C} \Lambda$, обладающих свойствами $\mathrm{C}^{\mathrm{T}} \mathrm{C}=\mathrm{I}, \mathrm{CC}^{\mathrm{T}}=\mathrm{I}$, $\Lambda_{66}=\operatorname{diag}\left(\lambda_{1, \ldots,} \lambda_{6}\right)$,

$\operatorname{trace}\left(\mathrm{R}_{66}\right)=\operatorname{trace}\left(\Lambda_{66}\right)=$

$(1.8076+1.7936+1.1509+0.8555+0.2887+0.1037)=6$.

В работах [2,3] эта задача формулируется как прямая спектральная задача (ПС3): $\mathrm{R}_{66}=>\left(\mathrm{C}_{66}, \Lambda_{66}\right)$ - прямая задача диагонализации известной выборочной корреляционной матрицы $\mathrm{R}_{66}=\mathrm{R}^{\mathrm{T}}{ }_{66}$, решаемая для известной симметрической матрицы $\mathrm{R}_{66}$, в результате решения которой вычисляются 2 матрицы: ортогональная матрица $\mathrm{C}_{66}$ собственных векторов $\mathbf{c}_{\mathbf{j}}=\left(\mathrm{c}_{1 \mathrm{j}}, \mathrm{c}_{2 \mathrm{j}} \ldots \mathrm{c}_{6 \mathrm{j}}\right)^{\mathrm{T}}$, расположенных по её столбцам: $\mathrm{C}_{66}=\left[\mathrm{c}_{1}\left|\mathbf{c}_{2}\right| \ldots \mid \mathbf{c}_{6}\right]$, согласованная со спектром $\Lambda_{66}=\operatorname{diag}\left(\lambda_{1}, \ldots \lambda_{6}\right)$ таким образом, что $\mathrm{RC}=\mathrm{C} \Lambda, \quad \mathrm{C}^{\mathrm{T}} \mathrm{C}=\mathrm{CC}^{\mathrm{T}}=\mathrm{I}_{66}$, $\operatorname{diag}\left(\mathrm{R}_{66}\right)=(1, \ldots, 1), \operatorname{tr}\left(\mathrm{R}_{66}\right)=1+1+\ldots+1=\operatorname{tr}\left(\Lambda_{66}\right)=\lambda_{1}+\ldots$ $+\lambda_{6}=6, \lambda_{1} \geq \ldots \geq \lambda_{6} \geq 0$. Выше в статье в таблице 2 приведены единственные решения $\mathrm{C}_{66}$ и $\Lambda_{66}=(1.8076, \quad 1.7936, \quad 1.1509, \quad 0.8555,0.2887$, 0.1037) Если ПСЗ имеет одно решение $\mathrm{C}_{66}$, то OC3 (обратная спектральная задача): $\Lambda=>\left(\mathrm{C}^{(\ell)}\right.$,
$\left.\mathrm{R}^{(\ell)}\right), \ell=1, \ldots, \infty$, имеет бесконечное множество решений $\quad \mathrm{C}^{(\ell)}{ }_{66}$ таких, что $\mathrm{R}^{(\ell)}{ }_{66} \mathrm{C}^{(\ell)}{ }_{66}=\mathrm{C}^{(\ell)}{ }_{66} \Lambda_{66}, \mathrm{C}^{(\ell) \mathrm{T}}{ }_{66} \mathrm{C}^{(\ell)}{ }_{66}=\mathrm{C}^{(\ell)}{ }_{66} \mathrm{C}^{(\ell) \mathrm{T}}{ }_{66}=\mathrm{I}_{66}$.

Среди бесконечного множества матриц $\mathrm{C}^{(\ell)}{ }_{66}$ существуют такие, у которых компоненты $\mathrm{c}^{(\ell)} 11$, $\mathrm{c}^{(\ell)}{ }_{21}$ 1-го собственного вектора близки к значениям $\mathrm{c}_{11}=0.6529, \mathrm{c}_{21}=0.6379$, компоненты $\mathrm{c}^{(\ell)} 42, \quad \mathrm{c}^{(\ell)}{ }_{52} \quad 2$-го собственного вектора доминируют и близки к значениям $\mathrm{c}_{42}=0.514$, $\mathrm{c}_{52}=0.6940$, компоненты $\mathrm{c}^{(\ell)}{ }_{33}, \mathrm{c}^{(\ell)}{ }_{43}, \mathrm{c}^{(\ell)}{ }_{63} 3-$-го собственного вектора доминируют и близки к значениям $\mathrm{c}_{33}=0.4631, \mathrm{c}_{43}=-0.5614, \mathrm{c}_{63}=0.6637$, т.е. по абсолютному значению принадлежат интервалу $[0.5,0.7]$ «заметных» значений коэффициентов корреляций (по критерию Чеддока), а остальные компоненты по своим значениям принадлежат интервалам пренебрежимо малых значений коэффициентов корреляций. Это служит подтверждением статистической достоверности вычисленных значений рисков. Компьютерное моделирование с применением программы CORMAP [22] требует сотен миллионов обращений к ней за несколько десятков часов машинного времени - необходимо отдельное исследование. 


\begin{tabular}{|c|c|c|c|c|c|c|}
\hline Impact Factor: & $\begin{array}{l}\text { ISRA (India) } \\
\text { ISI (Dubai, UAE } \\
\text { GIF (Australia) } \\
\text { JIF }\end{array}$ & $\begin{array}{r}=1.344 \\
=0.829 \\
=0.564 \\
=1.500\end{array}$ & $\begin{array}{l}\text { SIS (USA) } \\
\text { PИНЦ (Russia) } \\
\text { ESJI (KZ) } \\
\text { SJIF (Morocco) }\end{array}$ & $\begin{array}{l}=0.912 \\
=0.207 \\
=\mathbf{3 . 8 6 0} \\
=\mathbf{2 . 0 3 1}\end{array}$ & $\begin{array}{l}\text { ICV (Poland) } \\
\text { PIF (India) } \\
\text { IBI (India) }\end{array}$ & $\begin{array}{l}=6.630 \\
=1.940 \\
=4.260\end{array}$ \\
\hline
\end{tabular}

Таблица «заметных» значений рисков ФИ и их «заменяющих» в 6 НФИ

Таблица 4

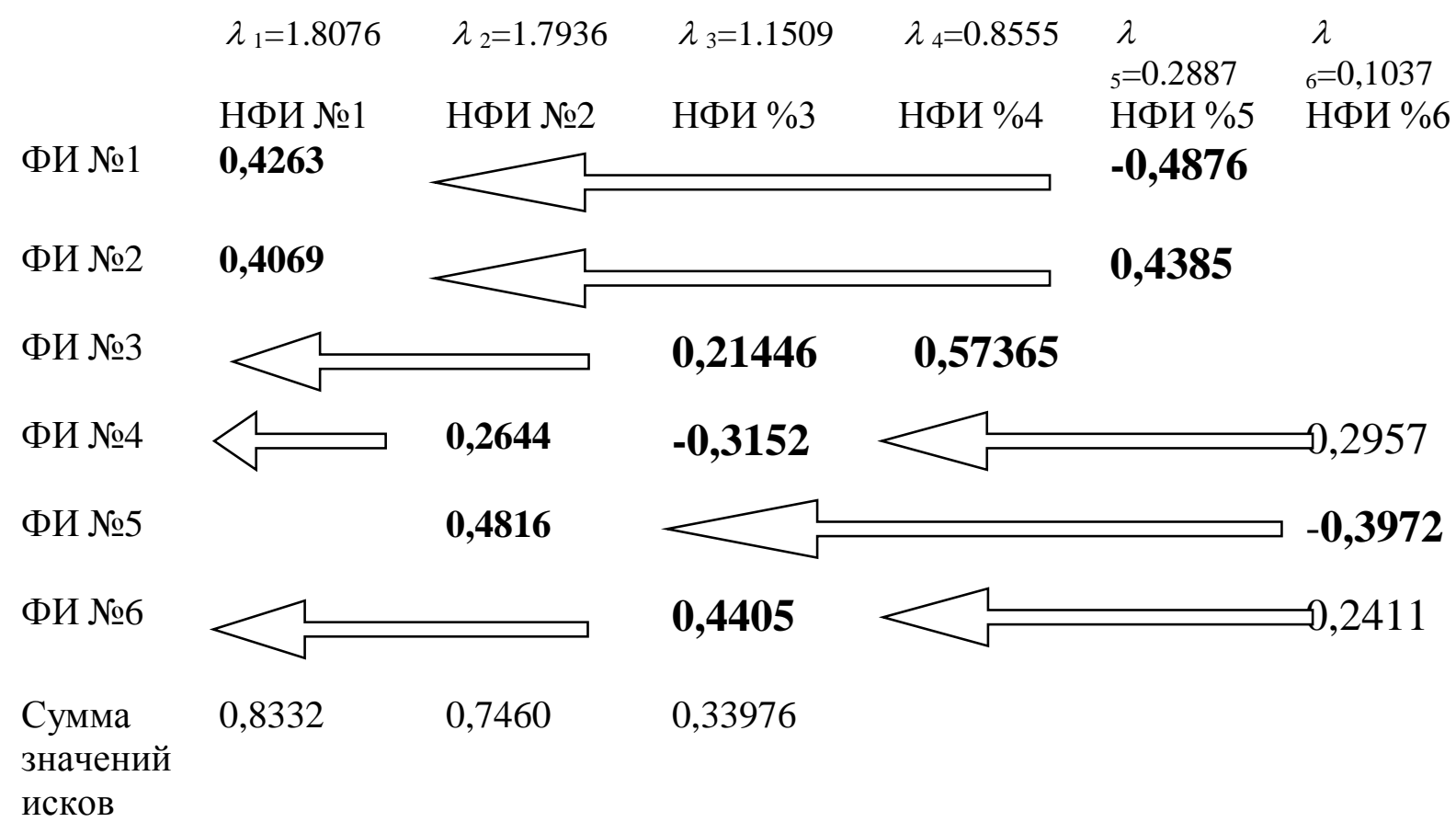

Применяемая модель анализа главных компонент [1,2,3] адекватно моделирует случайные переменные, корреляционные связи, особенности изотермных рядов процентных ставок типа «доходность к дате погашения». Сравнение величин рисков ФИ и НФИ (до и после перехода к некоррелированным $\mathrm{y}$ переменным) показывает (Рисунок 2) меньшую крутизну уменьшения значений $\lambda_{\mathrm{j}} / 6, \mathrm{j}=1, \ldots, 6$, рисков НФИ (на Рисунке 2 изображены значения $\lambda_{\mathrm{j}}$ ) по сравнению с крутизной

уменьшения значений рисков ФИ при удлинении сроков жизни ФИ. Переход к НФИ увеличивает значения и доли рисков более коротких ФИ. Не меняя сумму рисков ФИ. Степень рисковости НФИ №1 равна $\lambda_{1} / 6=1.8076 / 6=30.13 \%$,

НФИ№2 $-\lambda_{2} / 6=1.7936 / 6=29.89 \%$, НФИ№3 - $\lambda_{3} / 6=1.1509 / 6=19.18 \%$.

Степень рисковости НФИ № 1 и № 2 практически равны. Это были риски каждого из НФИ в отдельности. 


\begin{tabular}{|c|c|c|c|c|c|c|}
\hline Impact Factor: & $\begin{array}{l}\text { ISRA (India) } \\
\text { ISI (Dubai, UAE } \\
\text { GIF (Australia) } \\
\text { JIF }\end{array}$ & $\begin{array}{l}=1.344 \\
=0.829 \\
=0.564 \\
=1.500\end{array}$ & $\begin{array}{l}\text { SIS (USA) } \\
\text { PИНЦ (Russia) } \\
\text { ESJI (KZ) } \\
\text { SJIF (Morocco) }\end{array}$ & $\begin{array}{l}=0.912 \\
=0.207 \\
=\mathbf{3 . 8 6 0} \\
=\mathbf{2 . 0 3 1}\end{array}$ & $\begin{array}{l}\text { ICV (Poland) } \\
\text { PIF (India) } \\
\text { IBI (India) }\end{array}$ & $\begin{array}{l}=6.630 \\
=1.940 \\
=4.260\end{array}$ \\
\hline
\end{tabular}

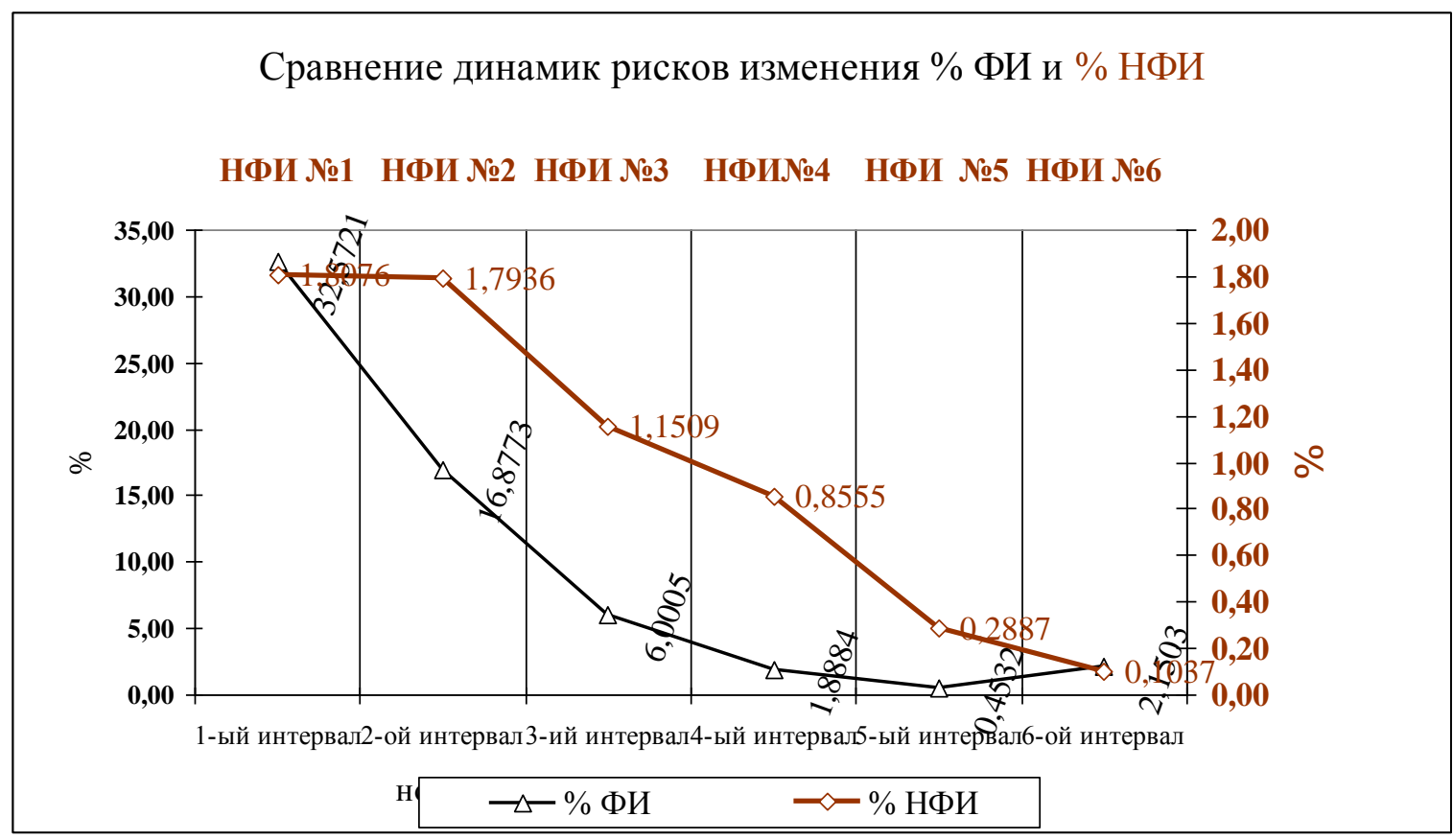

Рисунок 2 - Сравнение динамик вычисленных значений рисков изменения доходностей типа «доходность к дате погашения» ФИ и НФИ

\section{Заключение.}

С применением модели главных компонент получены новые кластеры (локальные портфели) высокорисковых (с относительно высокими доходностями) валютных ГЦБ имеющих распределение этих активов по степени рисковости изменения процентных ставок типа «доходность к дате погашения». Это позволило вычислить значения рисков изменения значений доходностей типа «доходность к дате погашения» НФИ №1 30.13\%,НФИ №2- 29.89\%, НФИ №3$19.18 \%$. Высокие степени извлекаемости значений рисков из коррелированной системы процентных ставок являются практическими критериями адекватности разработанной нами модели. В зависимости от склонности восприятия рисков дилеры банка будут покупать высокорисковые ГЦБ из НФИ №1: в нем выявлено (отражено в формулах) $83.32 \%$ рисков ФИ №1, ФИ №2, (и) или из НФИ №2: выявлено
$74.60 \%$ рисков ФИ №4, ФИ №5, и (или) из НФИ №3: в нем выявлено 33.976\% рисков ФИ №3, ФИ №4, НФИ №6. Все активы, независимо от уровня степени срочности были представлены в выделенных кластерах (НФИ). Это - новое «цифровое» доказательство типичности ажиотажа среди дилеров банков перед аукционами дневных торгов ГЦБ на фондовой бирже Республики Казахстан. Разработанная модель по новому классифицирует виды ГЦБ как активы банка: интервал №1 нашей временной структуры характеризует «короткие» ГЦБ, интервалы №2, №3, №4 - «краткосрочные» ГЦБ, интервал №5 - «среднесрочные» ГЦБ, №6«долгосрочные» ГЦБ. Это требует разработки нового приложения к бухгалтерскому балансу банка.

\section{References:}

1. Hotelling H. (1933) Analysis of a complex of statis tical variables into principal components. - J. Educ.Psychol., 1933, vol.24, pp.417-441, pp.498-520.
ISPC Technology and Innovation, Philadelphia, USA
2. Zhanatauov S.U. (1987) The inverse problem of the principal component analysis// Proc.of the 1-st World Congress of Soc. Math. Statist. and 


\begin{tabular}{|c|c|c|c|c|c|c|}
\hline Impact Factor: & $\begin{array}{l}\text { ISRA (India) } \\
\text { ISI (Dubai, UAE } \\
\text { GIF (Australia) } \\
\text { JIF }\end{array}$ & $\begin{array}{l}=1.344 \\
=0.829 \\
=0.564 \\
=1.500\end{array}$ & $\begin{array}{l}\text { SIS (USA) } \\
\text { PИНЦ (Russia) } \\
\text { ESJI (KZ) } \\
\text { SJIF (Morocco) }\end{array}$ & $\begin{array}{l}=0.912 \\
=\mathbf{0 . 2 3 4} \\
=\mathbf{3 . 8 6 0} \\
=\mathbf{2 . 0 3 1}\end{array}$ & $\begin{array}{l}\text { ICV (Poland } \\
\text { PIF (India) } \\
\text { IBI (India) }\end{array}$ & $\begin{array}{l}=6.630 \\
=1.940 \\
=4.260\end{array}$ \\
\hline
\end{tabular}

Pro babillity Theory of Bernoulli.-Utrecht,1987. - pp.116-119.

3. Zhanatauov S.U. (2013) Obratnaya model' glavnykh komponent.-Almaty:Kazstatinform, 2013. - $201 \mathrm{p}$.

4. FursovV.G. (2013) Innovatsionnaya napravlen nost' nauchnogo issledovaniya (retsenziya na monografiyu Zhanatauova S.U. «Obratnaya model' glavnykh komponent».-Almaty: Kazstatinform, 2013.-201p.).Vestnik KazNTU, №1, p.370-373.

5. (2015) Principal Component Analysis of Swap Curve Movements in Two Different Swap Markets: The Norwegian and Euro swap market. Norwe gian University of Life Sciences. Nordal, Simen Christoffer (Master thesis, 2015-08-06).

6. Pérignon,C., Smith,D.R.,Villa,C. (2007) Why common factors in international bond returns are not so common. Journal of International Money and Finance 26 (2007), 284-304.

7. Fase, M.G. (1973) A principal components analysis of market interest rates in the Netherlands, 1962-1970.European Economic Review,1973. Vol. 4, pp. 107-134.

8. Stelmach, J. (2010) Testing the homogeneous Interest Rates assumption by Principal Component Analysis:The Euro area case. Ekonomika, 2010. Vol. 89(3).

9. Lardic, S., Priaulet, P., Priaulet, S. (2003) PCA of yield curve dynamics: questions of methodologies. Journal of Bond Trading and Management 1, 2003. 327-349.

10. Lekkos, I. (2017) A critique of factor analysis of interest rates. Journal of Derivatives 8, 7273.

11. (1998) Finansy. Per. s angl. B. S. Pinskera «ZAO Olimp-Biznes», 1998.-560 p.: ill. Seriya «masterstvo».

12. (1996) Kolichestvennye metody finansovogo anna liza.(Professional'naya biblioteka, seriya «Finansy»)/pod red.BraunaS.,Kritsmena - M.: INFRA-M,1996. - 336 p.

13. WatshamT.,ParramoreK. QuantitativeMethods in Finance.Thomson Learning,1-st Edition.

14. Drobyshevskiy M.P. (2006) Obzor teoriy vremennoy struktury. M.: IEPP, 2006.-416 p.

15. SurkovG. (1999) Granitsy primenimosti metodo logii Value-at Risk dlya otsenki rynochnykh riskov: zarubezhnyy opyt i rossiyskaya praktika.Finansy,№10(199), 1999, p.59-65.

16. Kahn R.H. (1989) Risk and return in the US bond market: a multifactor approach. In F.J. Fabozzi (td.) Advances and innovations in the bond and Mortage Markets. Probus publishing, Chicago, 1989.

17. Kahn R.H. (1990) Estimating the US treasury term structure of interest rates. In F.J.Fabozzi (ed.). The Handbook of treasury and Goverentment Agency securities: Instruments, Strategies and Analysis. Probus publishing, Chicago. 1990

18. Kahn R.H., Guljarani D. (1993) Risk and return in the Canadian Bond Market. Journal of portfoljo Management., Spring 1993, Vol. 19, No. 3: pp. 86-93.

19. Zhanatauov S.U. (2016) Model and histogram to adequacy of variables $(\mathrm{C}, \Lambda)$-samples and real multidimensional sample. International Scientific Journal Theoretical\&Applied Science. 2016, № 11, vol. 43, pp. 53-61. Doi: http://dx.doi.org/10.15863/TAS.2016.11.43.11

20. Zhanatauov S.U. (2014) Teorema-kriteriy ravens- tva resheniy pryamoy i obratnoy zadach analiza glavnykh komponent. Evraziyskiy soyuz uchenykh.X mezhd.konf.«Sovrem. Kontsep tsii nauchnykh issled.». Moscow, 2730 dec. 2014. p. 55-58.

21. Zhanatauov S.U. (2013) The criterion of equality of solutions of the direct and inverse problems of the principal component analysis. «Seattle-2013 :4th International Academic Research Confe rence on Business, Education,Nature and Tech nology». 4-5 November 2013, p.447-449.

22. ZhanatauovS,U. (1988) O funktsional'nom napol nenii PPP “Spektr”./Sistemnoe modelirovanie - 13 .- Novosibirsk, 1988, p.3-11.

23. ChalmersC.P. (1975) Generation of correlation mat rices with a given eigen-structure. -J. Stat. Comp. Simul., 1975, vol.4, p.133-139.

24. Zhanatauov S.U. (2014) The (C, $\Lambda, Y)$-sample is adequate to real multidimensional sample/ Marep.Mezhdun.Konfer."Leadership in Educa tion, Business and Culture". 25 apriel 2014, Almaty-Seatle, ICET USA. Leadership Iternational Conference "Leadership on Education, Business and Culture». p.151-155.

25. Überla K. (1977) Factoren analyse. Eine systematische Einfuhrung in Psychologien, Mediziner, Wirtschafts-, und Socialwissenchaftler. 2 Auf lage. Berlin: Springer. Last edition 1977.

26. Horn J.L. (1965) A rationale and test for the number of factors in factor analysis. Psychometrika, 1965, vol.30, No 2, p. 179-185.

27. Jolllif I.T. (1972) Discarding variables in a principal component analysis. 1. Artificial data. -Appl. Stat., 1972, vol.21, p.160-173. 